Western University

Scholarship@Western

Brain and Mind Institute Researchers'

Publications

Brain and Mind Institute

$10-1-2013$

\title{
The touchscreen operant platform for assessing executive function in rats and mice.
}

Adam C Mar

Alexa E Horner

Simon R O Nilsson

Johan Alsiö

Brianne A Kent

See next page for additional authors

Follow this and additional works at: https://ir.lib.uwo.ca/brainpub

Part of the Neurosciences Commons, and the Psychology Commons

\section{Citation of this paper:}

Mar, Adam C; Horner, Alexa E; Nilsson, Simon R O; Alsiö, Johan; Kent, Brianne A; Kim, Chi Hun; Holmes, Andrew; Saksida, Lisa M; and Bussey, Timothy J, "The touchscreen operant platform for assessing executive function in rats and mice." (2013). Brain and Mind Institute Researchers' Publications. 101. https://ir.lib.uwo.ca/brainpub/101 


\section{Authors}

Adam C Mar, Alexa E Horner, Simon R O Nilsson, Johan Alsiö, Brianne A Kent, Chi Hun Kim, Andrew Holmes, Lisa M Saksida, and Timothy J Bussey 


\title{
The touchscreen operant platform for assessing executive function in rats and mice
}

\author{
Adam C Mar ${ }^{1,2}$, Alexa E Horner ${ }^{1-3}$, Simon R O Nilsson ${ }^{1,2}$, Johan Alsiö ${ }^{1,2}$, Brianne A Kent ${ }^{1,2}$, Chi Hun Kim ${ }^{1,2}$, \\ Andrew Holmes ${ }^{4}$, Lisa M Saksida ${ }^{1,2}$ \& Timothy J Bussey ${ }^{1,2}$
}

\begin{abstract}
${ }^{1}$ Department of Psychology, University of Cambridge, Cambridge, UK. ${ }^{2}$ Behavioural and Clinical Neuroscience Institute, University of Cambridge, Cambridge, UK. ${ }^{3}$ Synome Ltd., Babraham Research Campus, Cambridge, UK. ${ }^{4}$ Laboratory of Behavioral and Genomic Neuroscience, National Institute on Alcohol Abuse and Alcoholism (NIAAA), US National Institutes of Health, Bethesda, Maryland, USA. Correspondence should be addressed to A.C.M. (am682@cam.ac.uk).
\end{abstract}

Published online 19 September 2013; doi:10.1038/nprot.2013.123

This protocol details a subset of assays developed within the touchscreen platform to measure various aspects of executive function in rodents. Three main procedures are included: extinction, measuring the rate and extent of curtailing a response that was previously, but is no longer, associated with reward; reversal learning, measuring the rate and extent of switching a response toward a visual stimulus that was previously not, but has become, associated with reward (and away from a visual stimulus that was previously, but is no longer, rewarded); and the 5-choice serial reaction time (5-CSRT) task, gauging the ability to selectively detect and appropriately respond to briefly presented, spatially unpredictable visual stimuli. These protocols were designed to assess both complementary and overlapping constructs including selective and divided visual attention, inhibitory control, flexibility, impulsivity and compulsivity. The procedures comprise part of a wider touchscreen test battery assessing cognition in rodents with high potential for translation to human studies.

\section{INTRODUCTION}

Executive function can be conceptualized as a set of processes or mechanisms that coordinate and regulate other cognitive (sub)processes or behaviors. Although there is as yet no precise formal definition of what executive functioning entails, it is generally thought to contribute importantly to the dynamics and organization of flexible, goal-directed behavior and to be distinguishable from other basic cognitive properties (e.g., memory and perception) that it governs $s^{1,2}$. Executive function is typically described as a collection of processes that includes selecting, updating and planning motor sequences; withholding and stopping actions; monitoring and changing behavior where appropriate; and dividing, switching and sustaining attention ${ }^{3-5}$. These processes are likely to share overlapping features and mechanisms while also representing distinct psychological constructs ${ }^{3,6}$. The disruption of such processes is associated with a variety of psychiatric and neurological disorders ${ }^{7-9}$, and there is considerable evidence suggesting that executive function depends, at least in part, on the functional integrity of the frontal lobes $10-12$. It is thus increasingly important to establish preclinical assays of executive function in rodents that are standardized, reliable, practical (e.g., easy to implement) and translational, providing valid (e.g., face, predictive and construct) models of cognitive processes in humans (for recent discussions of translational validity in animal models of human cognition, see refs. 13-16).

Numerous paradigms and procedures have been designed to measure aspects of executive function. In humans, prototypical examples include the Wisconsin card sorting test, the Tower of London test, the spatial working memory task, the Stroop task, reversal learning paradigms and the continuous performance test ${ }^{17-21}$. In nonhuman primates and rodents, numerous analogs to these tests have been developed, such as odor- and visual-based intradimensional or extradimensional set-shifting tasks, as well as maze and operant chamber variants of tests of spatial working memory, reversal learning and attentional control ${ }^{22-26}$. This article focuses on three appetitive procedures that have been developed within the rodent touchscreen platform to assess certain aspects of executive function: extinction, an assay of the rate and extent of curtailing (inhibiting) a learned response that was previously, but is no longer, associated with reward; reversal learning, an index of the rate and extent of switching a response toward a visual stimulus that was previously not, but has become, associated with reward (and away from a visual stimulus that was previously, but is no longer, rewarded); and the 5-CSRT task, which measures the ability to selectively detect and appropriately respond to briefly presented, spatially unpredictable visual stimuli. These three tests have undergone validation work and have proven useful for assessing aspects of executive function across a variety of animal models. However, our laboratory and others are working to continually improve the current protocols as well as to develop new translational tests to assess executive function.

Strengths and weaknesses of the protocol

As indicated below, each of the three tasks described in the present protocol has also been implemented in non-touchscreen testing apparatus such as experimental mazes or specialized operant chambers. However, there are several advantages to using the touchscreen method for assessing cognition in rodent, as has been described previously ${ }^{27-30}$. In brief, these include a high degree of automation and standardization, the ability to test numerous subjects simultaneously, the minimization of possible confounds and variability due to within- or between-trial handling, the use of similar stimulus and response characteristics to those used by analogous tasks in humans and non-human primates, the capacity to introduce novel visual elements and the ability to crosscompare a behavioral test against a battery of cognitive tasks within identical apparatus in order to help delineate factors contributing to test performance or the effects of an experimental manipulation.

Relative weaknesses of the method include moderate-to-long training procedures, potential confounds due to disruptions 
or differences in subjects' visual functioning, as well as limitations common to most appetitive, operant paradigms (e.g., performance may be sensitive to variations in food restriction, hedonic/motivational factors, basic learning mechanisms and motor control). Importantly, the effect of several of these latter confounds can be assessed and potentially ruled out by running appropriate control experiments or by inspecting relevant dependent variables such as trial omissions and reaction times to elicit a response or to collect rewards. Moreover, the ability to test many animals in parallel may serve to offset any detriment resulting from extended training periods.

\section{Extinction}

The ability to learn to stop making a response that no longer provides a desired or adaptive outcome can be just as important as learning to produce the behavior in the first place. Since early descriptions of extinction ${ }^{31}$, it has become clear that it is a complex phenomenon: in many instances, behavioral changes cannot be simply explained by forgetting what has previously been learned, and its expression has been demonstrated to be highly sensitive to context ${ }^{32,33}$. Much of the recent literature on extinction learning has been devoted to examining the mechanisms underlying extinction of conditioned fear responses, typically using standard operant testing apparatus ${ }^{34,35}$. Although fewer studies have investigated the neural basis of extinction of learned appetitive responses, there is evidence that it involves, at least to some extent, similar substrates as fear extinction. Ventromedial regions of the prefrontal cortex (PFC) in humans and infralimbic cortex in rodents, as well as relevant subcortical circuitry including the striatum and amygdala, have been implicated in extinction of appetitive responding 36,37 . Recent reports have further suggested that abnormal resistance to extinction of appetitive responses may, in part, underlie disorders characterized by compulsive behavior, such as addiction and obsessive compulsive disorder ${ }^{37,38}$. Thus, extinction learning may be aligned with executive functioning through its parallels with other forms of inhibitory control, its theoretical complexity and its modulation by specific frontal brain circuitries.

In the extinction procedure outlined here, animals are first trained to acquire a simple visually guided response (e.g., touch a white square) in order to earn a reward. After this acquisition phase, animals are presented with similar opportunities to respond, but in the absence of rewards and associated cues. The time it takes and the extent to which animals suppress their responding provide the basic indices of extinction learning. Variants of this protocol have been used to test extinction in various mutant mouse lines ${ }^{39-43}$ and genetic strains ${ }^{44,45}$. We have only recently started using this procedure in rats, and no major issues have been observed or are expected in translating this relatively straightforward task. Indeed, quite similar methods for testing extinction can be used in most operant settings in which rats or mice have learned to make a response for reward ${ }^{46,47}$.

Touchscreen extinction assays have been effectively used to phenotypically characterize behavioral differences between inbred mouse strains. These studies have shown that some strains of mice, including the commonly used $\mathrm{C} 57 \mathrm{BL} / 6 \mathrm{~J}$ and $\mathrm{BALB} / \mathrm{cJ}$ mice, perform well on the extinction task compared with certain other strains, such as DBA/2J mice. In addition, some mouse strains, such as $129 \mathrm{~S} 1 / \mathrm{SvImJ}$ have been found to show normal touch- screen extinction despite being markedly impaired on other (e.g., Pavlovian fear) measures of extinction ${ }^{44}$. Although the principal brain regions mediating touchscreen extinction have not yet been identified using lesion or other techniques, studies using genetic mutant mice have provided preliminary insights into the molecular basis of the behavior. For example, deletion of a gene encoding one major glutamate receptor subtype, AMPA GluA1, substantially retarded extinction ${ }^{41}$, whereas neither deletion of the gene encoding the NMDA receptor subunit GluN2A (ref. 39) nor the gene encoding the glutamate transporter GLAST ${ }^{40}$ disrupted the behavior. Moreover, deletion mutations of two paralogs of the discs, large homolog (Dlg) family of postsynaptic scaffold proteins resulted in reciprocal effects: extinction was retarded in $\mathrm{Dlg} 2^{-1-}$ mice but significantly enhanced in $\mathrm{Dlg} 3^{-1-}$ mice ${ }^{42}$. Enhanced extinction has also recently been observed in the TgCRND8 mouse model of Alzheimer's disease (AD)-related amyloid pathology ${ }^{43}$. These findings highlight the bidirectional sensitivity of the touchscreen extinction assay and the utility of a test battery approach using multiple touchscreen tasks ${ }^{29}$, in which effects on extinction can be distinguished from, and otherwise inform, effects on other executive functions.

Although the touchscreen extinction assay described here is relatively simple to implement, extinction is a complex phenomenon with numerous factors or underlying processes that putatively contribute to its expression (e.g., generalization decrement, response inhibition, Pavlovian and/or instrumental learning mechanisms $)^{48}$. On the one hand, this cautions that care must be taken when interpreting extinction results, particularly with respect to an animal's distant and recent learning historyextinction has been noted to be highly dependent on context. On the other hand, it suggests that there are a variety of behavioral probes, modifications and extensions of the current procedure (e.g., reinstatement, renewal and reacquisition) that can provide valuable additional insights ${ }^{45}$. The flexibility and breadth of stimulus control offered by the touchscreen platform make it particularly well suited for innovating and expanding research examining extinction processes.

\section{Reversal learning}

In addition to extinction learning, organisms need to be able to flexibly adjust their behavior in other ways when faced with changing environments or rules. Appetitive reversal learning procedures are widely used assays for such flexibility. In these procedures, subjects are first taught to discriminate and choose a rewarded over an unrewarded stimulus or response option. After this discrimination learning phase, the reward associations are switched and subjects must learn not only to extinguish the previously rewarded response but also to choose the previously unrewarded (now-rewarded) option. The rate and extent to which the new reversed discrimination is learned provides an index of flexibility. Reversal learning deficits have been observed in many neuropsychiatric disorders, including schizophrenia ${ }^{49}$, Parkinson's disease ${ }^{50}$ and obsessive-compulsive disorder ${ }^{51}$. There is also considerable evidence, across several different species, testing apparatus and protocols, linking reversal learning to a functional neural circuitry including the PFC-particularly orbitofrontal regions $\mathrm{s}^{23,52-58 \text { - and dorsal striatum }}{ }^{59}$ - as well as to neuropharmacological factors such as serotonin (5-HT) ${ }^{60-62}$ and dopamine signaling ${ }^{63-65}$. 
Related findings have also been observed using the rodent touchscreen visual reversal learning procedure, which has the translational advantage of incorporating near-identical stimulus and response characteristics to methods commonly used in humans and nonhuman primates. Orbitofrontal lesions in both rats $^{66,67}$ and mice ${ }^{68}$ substantially retard visual reversal learning, as do lesions of the dorsolateral striatum in the mouse ${ }^{68}$. Lesions of medial PFC in rats ${ }^{69}$ and mice ${ }^{39}$ have also been observed to impair reversal, but only when visual stimuli are difficult to discriminate. In contrast, amygdala lesions in rats ${ }^{67}$ and ventromedial-specific PFC lesions in mice were found to significantly facilitate reversal learning 68 . Touchscreen reversal learning has further been found to be retarded after systemic treatment with methamphetamine ${ }^{70}$, a D1-agonist ${ }^{71}$, as well as in NMDA receptor subunit GluN2Aknockout transgenic mice ${ }^{39}$. Enhanced reversal learning has been observed in the TgCRND8 mouse model of amyloid pathology 43 and after manipulations that elevate brain 5-HT content such as 5-HT transporter knockout or subchronic treatment with the serotonin-selective reuptake inhibitor, fluoxetine ${ }^{72}$. Deletion of the gene encoding the AMPA receptor subunit GluA1 also slightly improves reversal ${ }^{41}$.

As with extinction, these findings highlight the bidirectional sensitivity of the touchscreen visual reversal learning procedure. Moreover, there are additional processes that are thought to contribute to reversal beyond those described for extinction of a previously rewarded but currently unrewarded response option. These include overcoming the avoidance of a previously unrewarded response option, attending and selecting the appropriate response strategy (e.g., choosing on the basis of visual discrimination of stimuli rather than an egocentric position bias) and acquiring a new stimulus-reward association ${ }^{61}$. The flexibility of stimulus control offered by the touchscreen platform makes it particularly well suited for attempting to dissociate such processes (e.g., through introduction of the novel replacement stimuli and/or new response options; see Experimental design for further discussion).

\section{5-CSRT task}

The touchscreen 5-CSRT task is a modified version of the fivechoice task originally developed for rats using the classic ninehole operant chamber ${ }^{26}$, an analog of the 5-CSRT task used to study human attentional processes ${ }^{73}$. The operant chamber method has also been modified for use in mice ${ }^{74,75}$, and a considerable amount of work has now assessed 5-CSRT phenotypic differences between various mouse strains as well as in transgenic and mutant lines ${ }^{76-82}$.

The 5-CSRT task trains rodents to report the occurrence and location of brief, visual stimuli presented pseudorandomly across five spatial locations in a horizontal array of apertures. The task can be used to evaluate various aspects of executive function: response accuracy (proportion of correct over all attempted trials) is typically interpreted as a measure of sustained, spatially divided attention; omissions (trials when no response is made) are a putative index of global attentional processes; premature (responses before stimulus onset) or perseverative responses (extra responses after outcome feedback) are measures of inhibitory control, possibly related to the constructs of impulsivity and compulsivity, respectively. Additionally, response and reward collection latencies relate to processing speed and to motoric or motivational factors. All of these measures may reflect, to some extent, dissociable cognitive processes, and they have been demonstrated to be sensitive to distinct pharmacological treatments and the integrity of distinct sectors of the rodent PFC. Detailed summaries of the application and protocol of the traditional operant box versions of the 5-CSRT task have been previously described for both rats $^{83}$ and mice 75 .

The 5-CSRT task procedure outlined here was first developed by Susan Bartko, Carola Romberg and colleagues for testing mice using the touchscreen platform ${ }^{84,85}$. The touchscreen method has recently been used to examine executive function within various genetic mouse models. Relative to their wild-type controls, both a triple-transgenic $\mathrm{AD}$ mouse model ( $3 \times \mathrm{TgAD}$; ref. 84$)$ and the TgCRND8 mouse model of AD-typical amyloid pathology 43 showed decreased response accuracy across the session when the attentional load of the task was increased by shortening the duration of the target stimulus. The attentional deficits in $3 \times \mathrm{TgAD}$ mice could be ameliorated by administration of the cholinesterase inhibitor donepezil (Aricept), which has procognitive effects in AD patients. Mice with homozygous deletion of the gene encoding the cholinergic M1 receptor $\left(\mathrm{Chrm}^{-/-}\right)$showed no alterations in performance accuracy but were found to show lower omissions, higher premature responses and greater perseveration compared with wild-type mice ${ }^{85}$. More recently, it was found that inbred BTBR $\mathrm{T}+\mathrm{tf} / \mathrm{J}$ mice (a putative mouse model for autism) show decreased accuracy for detection of short stimuli as well as increased impulsivity and decreased motivation relative to C57Bl/6 J mice ${ }^{86}$. This method shares similar benefits and limitations as the traditional version ${ }^{83}$, with the additional advantages of superior flexibility and control over the visual target stimuli (e.g., brightness, contrast, size and spacing) and the ability to run the test in the same apparatus and behavioral setting as many other tests of cognition 30,87 .

\section{Experimental design}

General considerations. As the procedures in this protocol have primarily been published using mice as subjects, the main task descriptions are based on procedures developed for the mouse. For reversal learning, which was first developed in the touchscreen using rats ${ }^{69}$, the major differences between mouse and rat protocols are highlighted. Although extinction and 5-CSRT task touchscreen methods have not yet been published using rats, unpublished studies recently conducted in our laboratory suggest that no major issues are to be expected with translating these relatively straightforward and well-established tasks (K. McAllister, L. Lyon, A.C.M., L.M.S. and T.J.B., unpublished data). It should also be noted that, although the protocols described below are based on the original publications cited above, certain minor refinements have since been made to further optimize the procedures. Furthermore, this protocol describes the standard procedures as currently used in our laboratory.

In all of the tasks described here, the specific research question and experimental manipulation may affect the behavioral procedure. For clarity, we will limit discussion to four frequently used treatment scenarios. In case 1, the subject receives treatment before onset of the experiment (e.g., constitutive transgenic or knockout models and developmental manipulations). In case 2, the subject receives treatment before task acquisition but after pretraining (e.g., subchronic drug treatment and neurotoxic lesions). In case 3 , the subject receives treatment after acquisition to assess effects on asymptotic performance level or 
postacquisition behavioral challenges using a between-subject design (e.g., neurotoxic lesions and subchronic drug treatment). In case 4 , the subject receives a transient manipulation at performance level, or during postacquisition behavioral challenges, that can be performed with a within-subject design (e.g., systemic pharmacological or intracerebral infusion procedures). We will refer to these cases as appropriate in the procedure. If the effects upon both acquisition and the main task are of interest, the manipulation can be made before the start of the training (e.g., cases 1 and 2). However, if the effect on the main task is of primary or exclusive interest, the researcher may prefer to introduce the manipulation after the acquisition phase if it is practical to do so (e.g., cases 3 and 4). It should also be noted that although the 5-CSRT task typically yields stable levels of performance and lends itself well to examination of acute manipulations (e.g., case 4), animals typically require multiple sessions to achieve criterion in extinction and reversal learning (see TIMING), and it may be difficult to assess the effects of acute manipulations on learning in these procedures.

There are also several options for determining the point at which animals can be advanced from acquisition training to postacquisition manipulations (including cases 3 and 4, and postacquisition behavioral challenges including reversal learning, extinction and 5-CSRT probe phases):

- Fixed training period. Train all animals on a predetermined number of acquisition phase sessions before moving on to primary task phase or probe testing, regardless of the performance level. This protocol is desirable when it is essential for all animals to be reversed at a particular time point or age, such as when assessing progressive disease models ${ }^{88}$. However, by using this approach, some animals may receive substantial overtraining, whereas others may fail to acquire the initial contingencies, which may confound interpretation of subsequent primary task or probe test effects.

- Group criterion. Before proceeding to the primary task phase or probe tests, continually train animals on acquisition-phase training (e.g., pretraining phases and/or main task phase) until all animals have reached criterion. This protocol is desirable when complete acquisition curves are required, as all animals will have the same number of training days before the primary task or probe tests. However, some subjects might be overtrained and/or will have obtained more rewards during the acquisition phase, and this may confound performance in the primary task or probe tests.

- Individual criterion. Move each animal onto the primary task phase or probe tests on the session immediately after it has reached acquisition phase criterion. This option prevents possible carry-over effects due to overtraining during acquisition (extinction and reversal learning can be particularly sensitive to such overtraining). However, as the primary task testing is likely to be staggered across animals, decisions based on group-average performance levels can be difficult to make. Moreover, depending on how long the acquisition phase lasts for some animals, there may be marked time/age differences between individual subjects or experimental groups.

- Hybrid approach. This is a hybrid of the group criterion and individual criterion approaches. For most common experimental designs, we generally recommend this option. By using this option, animals are trained to criterion on all acquisition phases, and then put 'on rest' until all animals have reached criterion. Thereafter, before proceeding to the primary task phase or probe tests, all animals can be tested at the same time for a further one to three sessions to ensure they are all still performing at criterion. If there is a large discrepancy between the fastest and slowest learners during acquisition, it might be useful to run occasional 'reminder' sessions for the animals who have already reached criterion (e.g., one session per week). If an animal's performance falls below criterion in a reminder session, that animal is trained daily until criterion is reattained. This protocol option ensures that all animals experience their initial primary task phase session on the same test day and minimizes overtraining effects. However, depending on the variability of acquisition, it should be noted that some subjects may be exposed to many more sessions and rewards.

The three tests measuring the aspects of executive function described in this protocol may be administered together and/or as part of a flexible test battery that includes touchscreen tasks assessing other cognitive domains ${ }^{30,87}$. This battery approach can be tailored to test specific hypotheses or used to generate general cognitive profiles for individuals or groups of animals, which may aid in the interpretation of the behavioral data. The approach may be even more powerful if animals are tested within subjects (each animal is tested on all tasks of interest); however, care must be taken when determining an appropriate sequence of task presentation.

Pretraining. All three tasks in this protocol are motivated by food reward and require instrumental responses to the touchscreen. To ensure sufficient levels of motivation, animals thus are subject to mild food restriction beginning before pretask training, and this is continued throughout the course of the experiment. The purpose of pretraining is to gradually shape appropriate screen-touching behavior; it normally comprises three to five stages followed by training specific to each task. The number and size of response windows and the size and type of visual stimuli used during pretraining is task dependent. For the extinction and 5-CSRT tasks, the pretraining stimulus is a solid white square. For reversal learning of visual discrimination, pretraining stimuli are typically selected from a library of 40 varied black and white shapes, which are distinct from the stimuli used in the main task procedure. The rationale for using a large number of varied stimuli is to try to minimize generalization between the pretraining- and taskspecific stimuli.

After commencement of mild food restriction, animals are habituated to the food reward and chambers for at least two daily sessions (stage 1, see Steps 4 and 5). In stage 2 (Fig. 1), a series of trials are delivered in which a stimulus is presented within one of the response windows. If the stimulus is not touched within $30 \mathrm{~s}$, the stimulus is removed and its offset is concurrent with reward delivery and with activation of the magazine light and a brief $(1-\mathrm{s}, 3-\mathrm{kHz})$ tone (conditioned reinforcers). Touches to stimuli are encouraged by immediate offset of the stimulus, a triple reward delivery and activation of the magazine light and a tone. After reward retrieval, an intertrial interval (ITI) begins, after which the next trial is automatically initiated. For extinction and the 5-CSRT task, the ITI is typically set at $5 \mathrm{~s}$. For reversal learning, the ITI is typically set at $20 \mathrm{~s}$, as it has been demonstrated that longer ITIs might help facilitate learning 28 . Our current standard procedure is 
Figure 1 | Flowchart overview of pretraining stages 2-5. Stage 2: a visual stimulus is presented in one of the response windows. If it is not touched, stimulus offset occurs after $30 \mathrm{~s}$ and a reward is delivered. If it is touched, offset is immediate and a triple reward is delivered. After reward collection and an ITI period, the next stimulus is presented in a new trial. Stage 3 proceeds as in stage 2, but the stimulus remains on the touchscreen until touched. Animals to be tested on extinction can proceed directly to the extinction procedure after reaching criterion on stage 3 . Stage 4 proceeds as in stage 3 , but the animal must enter and exit the magazine after the ITI to initiate the next trial. Stage 5 proceeds as in stage 4, but touches to blank response windows (when there is a stimulus on the screen) are discouraged with a time out. After this and the ITI, the next trial may be initiated, but in pretraining for the majority of tasks this is a CT in which the previous stimulus is represented, rather than a new trial. Note that CTs are not given in stage 5 of pretraining for the 5 -CSRT. The labels in italics indicate steps in which the animal is required to perform an action.

to have the house light off during stimulus presentation and ITIs (and on for timeout periods; see stage 5 below), but each of these tasks have also been performed with the house light on. Although we have not made any direct comparisons between these procedural variants, there may be some reduction in the luminance contrast when the house light is on during stimulus presentation due to the higher ambient light levels, which may increase the threshold for signal detection.

Stage 3 (Fig. 1) is similar to stage 2 in all respects, with the exception that subjects are required to touch the stimulus to cause reward delivery, stimulus offset and activation of the magazine light and tone. Subjects proceeding to extinction training would do so after completing stage 3 .

Stage 4 (Fig. 1) is similar to stage 3, but subjects are further required to trigger stimulus presentation, referred to as trial 'initiation'. The session begins with a 'free' reward delivery and magazine illumination, indicating that a trial may be initiated by the subject. When the animal nose pokes into the magazine, the magazine light is extinguished and a brief (0.2-s) click sound is generated. When the animal withdraws its nose from the magazine, the stimuli are presented on the screen. Initiation is also required after the ITI between each trial.

Stage 5 (Fig. 1) is similar to stage 4, but subjects are additionally discouraged from touching blank response windows during stimulus presentation, via the negative feedback of immediate stimulus removal and initiation of a time-out period in which the house light illumination status is inverted (e.g., on-to-off or off-on; see stage 2 above). This stage serves to introduce the subject to the cue signaling incorrect responses (e.g., the time-out period). Only after the time-out period elapses does the ITI begin, after which the next trial can be initiated. For subjects proceeding to reversal learning training, any trial immediately following a time-out period is designated as a correction trial in which the same stimuli are represented in the same location(s). There is no limit on the number of correction trials that can be given
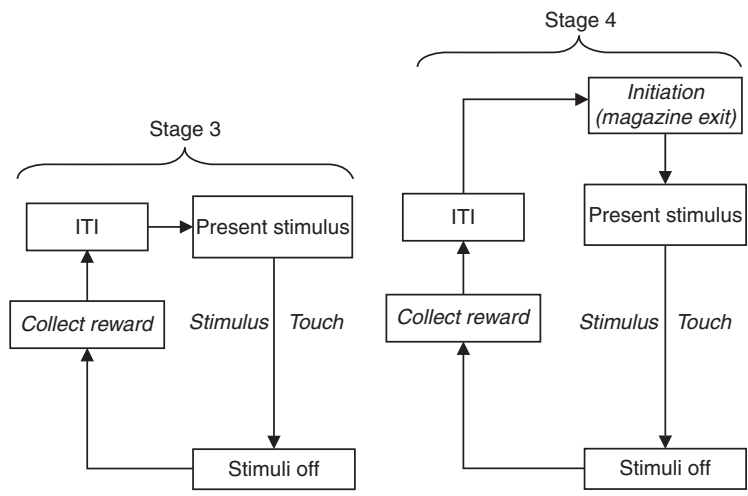

tage 5

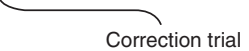

Normal trial Correction tria

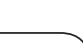

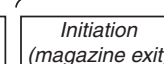

(magazine exit)
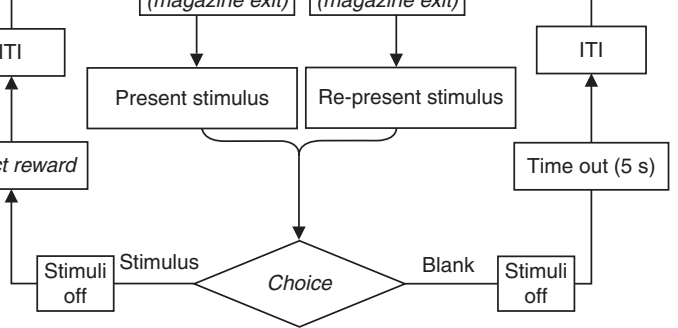

consecutively, but once the subject responds correctly, the correction procedure ends and a normal, noncorrection trial resumes. Correction trials do not count toward the session trial limit. The purpose of correction trials is to help counteract development of side or stimulus biases and to ensure that subjects receive a consistent number of rewards per session. Although correction trials may also be implemented for 5-CSRT task training, our current standard protocol does not include correction trials in 5-CSRT pretraining or in its main procedure.

By the end of stage 5 of pretraining, subjects should be completing a sufficient number of trials per session (as specified in the PROCEDURE) to promote the completion of sessions in the subsequent task. Note that rats are typically given the opportunity to complete more trials per session than mice (e.g., 100 as opposed to 30 during pretraining). Rats readily complete a greater number of trials per session than mice, perhaps because the mouse:rat body mass ratio is smaller compared with the mouse:rat reward pellet size ratio (14:45 mg).

Analysis of pretraining performance is generally minimal. The number of sessions required to complete each phase of pretraining, or the overall number of sessions required to complete pretraining, may be analyzed if desired or if you are matching for performance level before an experimental manipulation (see case 2 above).

Extinction. The extinction procedure described here is the product of a series of refinements to the methods described in recent publications (discussed above); changes have generally involved simplifying the stimulus and response requirements. The trial structure of the acquisition phase of the extinction procedure (Fig. 2) requires only a small modification of the standard operant pretraining paradigm (compare to pretraining stage 4 above). 

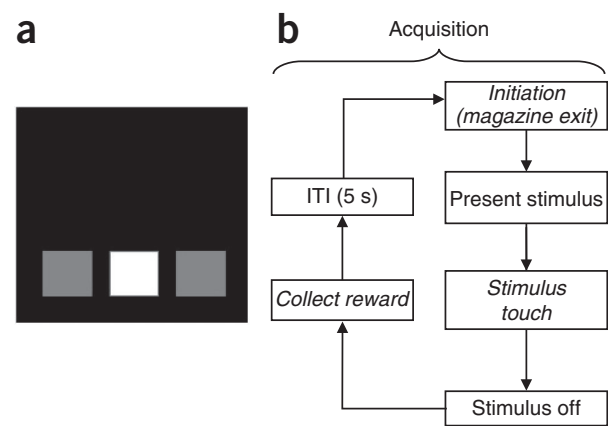

C

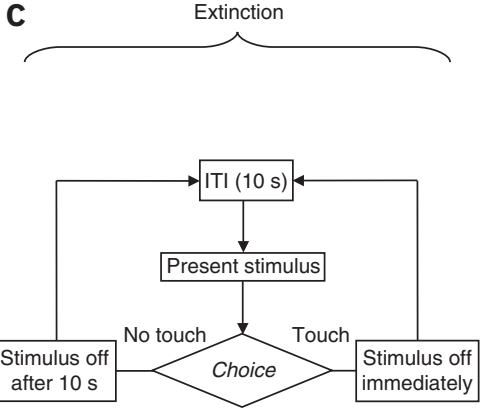

d

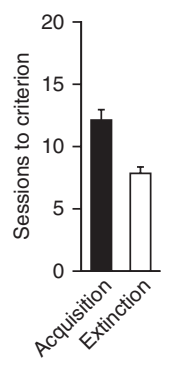

e

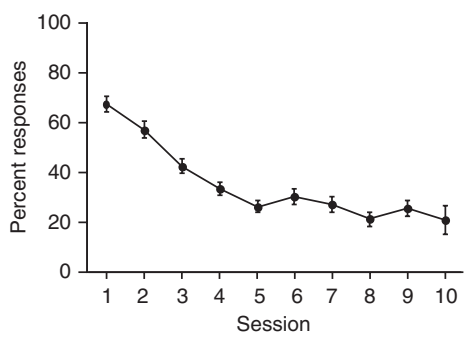

Figure 2 | Extinction task. (a) Schematic of a three-hole mask used in the extinction paradigm. (b) Flowchart overview of acquisition learning phase. After initiation, a solid white square stimulus is presented at a central location on the touchscreen. When the subject touches the stimulus, a reward is delivered, and after reward collection and an ITI, a new trial may be initiated. The labels in italics indicate steps in which the animal is required to perform an action. (c) Flowchart overview of extinction learning phase. Each trial begins with a 10-s ITI, after which the single, solid white square stimulus is presented on the touchscreen. The subject is not required to initiate the trial. If the subject either touches the stimulus (response) or does not touch the stimulus within a 10-s duration (omission), the stimulus is removed and the 10-s ITI leading to the next trial is initiated. No rewards or conditioned reinforcers (e.g., tray light or tone associated with reward delivery) are delivered during the extinction phase. The labels in italics indicate steps in which the animal is required to perform an action. (d) Representative data of C57BL/6J mice using a two-choice acquisition and extinction procedure, showing the typical number of sessions to criterion (data re-plotted from ref. 45). (e) Representative data from the same mice showing the time course of response extinction. Data are presented as means \pm s.e.m.

During the response acquisition phase, sessions begin with a free reward delivery to the magazine and magazine light illumination, indicating that a trial may be initiated. Trials are initiated by the subject's head entry into the magazine (turning off the magazine light and activating a 0.2 -s auditory click), in which subsequent head withdrawal from the magazine initiates presentation of a single, solid white square stimulus at a central location on the touchscreen. When the subject touches the stimulus, the stimulus is removed from the screen, a reward is delivered and the magazine light and a 1-s tone are turned on. Following reward collection, the magazine light is extinguished and a 5-s ITI commences, after which the magazine light is illuminated and subjects are again given the opportunity to initiate a new trial.

There are several alternatives to the response acquisition phase trial structure described above. For example, the procedure may be simplified even further, eliminating the requirement for trial initiation (e.g., proceeding directly to the extinction phase below from pretraining stage 3 above). This would permit faster pretraining times and also share more procedural similarity with the extinction phase procedure described below. Another possibility would be to randomize the position of the stimulus across two or three screen locations (using a two- or three-stimulus mask; see Equipment Setup). The acquisition phase could be programmed such that touches to blank locations cause disappearance of the stimulus, turn on the house light and begin a time-out period during which animals are unable to initiate a new stimulus presentation (compare to pretraining stage 5 above). Indeed, an extinction procedure using two stimulus locations on the touchscreen has been used frequently with success ${ }^{39-41}$. Although it is more complex, this approach can help distinguish whether subjects are acquiring a specific response to the visual stimulus or simply approaching the touchscreen. Indeed, virtually any response-learning procedure may be used before extinction learning, with the proviso that the rate, extent and interpretation of extinction learning can vary depending on what is learned during response acquisition or earlier. It is important to reiterate that, as expression of extinction is highly dependent on the training context, comparisons of the rates of extinction learning should only be made in light of similar training histories.

In the extinction phase of the current procedure (Fig. 2c), each trial typically begins with a 10-s ITI, after which the single, solid white square stimulus is presented on the touchscreen. The subject is not required to initiate the trial. If the subject either touches the stimulus (response) or does not touch the stimulus within a 10-s duration (omission), the stimulus is removed and the 10-s ITI leading to the next trial is initiated. No rewards or conditioned reinforcers (e.g., tray light or tone associated with reward delivery) are delivered during the extinction phase.

As animals typically require multiple sessions to achieve criterion in extinction (see TIMING and ANTICIPATED RESULTS), it may be difficult to investigate the effects of acute experimental manipulations (an example of case 4). However, task parameters in the extinction phase may be adjusted (e.g., decrease the criterion number of trials and/or increase the session length) to help facilitate investigations of acute manipulations. Various posttraining probe tests may also be carried out after the extinction phase (e.g., reinstatement procedures ${ }^{8}$ ) to further assess aspects of extinction learning that may be amenable to acute experimental manipulations. The most well-established post-extinction probes include various forms of relapse-the reoccurrence of the relevant behavior learned before extinction training 32,89 . For example, reacquisition may be assessed by examining recovery of pre-extinction behavior after the unconditioned reinforcer (i.e., food reward) and/or conditioned reinforcers (i.e., magazine light and brief tone) are reintroduced in a manner fully contingent on presentation of, and the subject's response to, the visual stimulus (i.e., identical to the learning stage before removal of these reinforcers during extinction). If reacquisition is only partial (e.g., limited in time or number of reinforcers) or re-exposure to the unconditioned stimulus is not delivered in a manner contingent on previously learned stimuli or responses, the probe procedure is typically described as reinstatement ${ }^{45}$. Reinstatement may also be assessed after contingent or noncontingent exposure to only the conditioned reinforcer(s) (e.g., without the 
a

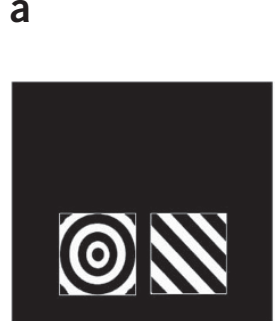

Figure 3 | Reversal learning task. (a) Schematic of a two-window mask and b

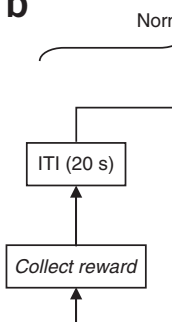
$\overbrace{}^{\text {Normal tria }}$
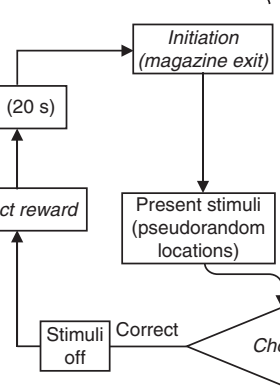
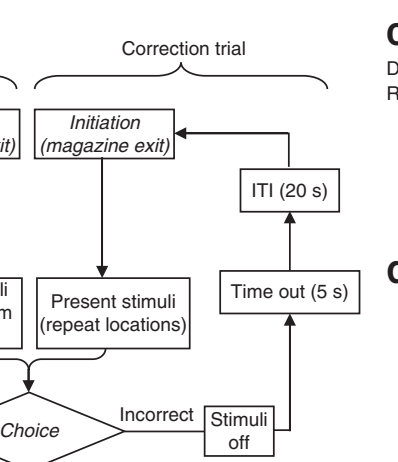

C
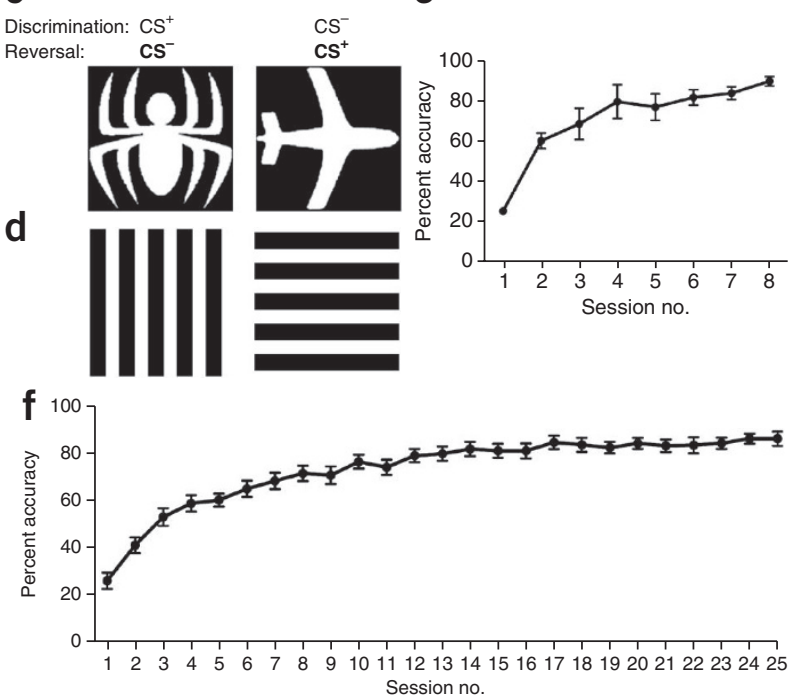

stimuli used in the reversal learning paradigm. (b) Flowchart overview of the reversal learning procedure. After initiation, a pair of stimuli $\left(\mathrm{CS}^{+}, \mathrm{CS}^{-}\right)$is presented on the screen, in pseudorandom locations. Correct responses (to $\mathrm{CS}^{+}$) are rewarded, and after reward collection and an ITI, a new trial may be initiated. Incorrect responses (to $\mathrm{CS}^{-}$) are discouraged with a time out, and then after an ITI and initiation the previous trial type is represented (a correction trial). The correction trial loop will continue until a correct response is made. The labels in italics indicate steps in which the animal is required to perform an action. (c) Spider versus plane stimuli typically used for visual discrimination and reversal learning in rats. (d) Horizontal versus vertical pattern stimuli, which rats acquire more readily. (e) Typical reversal learning performance in rats $(n=10$, with a history of object-location paired associates learning and trial-unique nonmatchingto-location training) using 'castle' versus 'face' photographic stimuli (C.A.0., unpublished data). (f) Typical reversal learning performance in mice ( $n=17$, of mixed background ( 1:15 CBA/ca:C57BL/6J), using 'marble' versus 'fan' stimuli (A.E.H., unpublished data). Data are presented as means \pm s.e.m.

unconditioned reinforcer), although such procedures that reintroduce elements of the pre-extinction learning context are more commonly referred to as renewal ${ }^{48,89}$. These postextinction probes may be used to evaluate propensity to relapse and also the nature and extent of extinction processes; for example, the strength of reinstatement is believed to be an indicator of the strength of contextual conditioning ${ }^{90,91}$. For a fuller theoretical discussion of such postextinction probes, see refs. 32,48.

The primary measures of extinction learning are the number of sessions, responses and trials required to reach criterion. In addition, response latencies and rates of responding can also be examined. Postextinction probe sessions are typically analyzed by calculating the differences between responses during the last extinction session(s) and those during the postextinction probe session(s).

Reversal learning. The trial structure of the visual reversal learning task requires a simple adjustment of the visual discrimination task (ref. 30) and is illustrated in Figure 3. Each session begins with a free reward delivery to the magazine and magazine light illumination, indicating that a trial may be initiated. A trial is initiated when the subject enters (signaled by a 0.2 -s auditory click and the magazine light turning off) and withdraws its head from the reward magazine; head withdrawal is followed immediately by the appearance of two stimuli in two distinct, predefined locations on the touchscreen (see Equipment Setup). One of the stimuli is designated as correct $\left(\mathrm{CS}^{+}\right)$, whereas the other is incorrect $\left(\mathrm{CS}^{-}\right)$. The location of the $\mathrm{CS}^{+}$and $\mathrm{CS}^{-}$is determined pseudorandomly for each trial, with the constraint that the stimuli are not displayed in the same locations for more than three consecutive trials (excluding correction trials). If the subject touches the correct stimulus, both stimuli are removed, the magazine light and a 1-s tone are turned on, and a reward is delivered. When the subject makes a magazine entry to collect the reward, a $20 \mathrm{~s}$ ITI is initiated. If the subject touches the incorrect stimulus, both stimuli are removed and the house light is turned on for a 5-s time-out period (no reward is delivered). When the time-out period has elapsed, the house light is switched off and the 20-s ITI is initiated. When the ITI period ends after either a correct or incorrect trial, the magazine light is turned on, allowing the opportunity for the subject to initiate a new trial. An incorrect trial causes the subsequent trial to be a correction trial, which is presented in an identical manner to the trials described above, with the constraint that the two stimuli are presented in same locations as the preceding trial. Correction trials continue to be presented until the animal touches the $\mathrm{CS}^{+}$, and they do not add to the total trial count for the session. Acquisition and reversal sessions of visual discrimination learning are identical, except that, during reversal learning, the previously correct stimulus $\left(\mathrm{CS}^{+}\right)$ becomes the incorrect stimulus $\left(\mathrm{CS}^{-}\right)$, whereas the previously incorrect stimulus $\left(\mathrm{CS}^{-}\right)$becomes correct $\left(\mathrm{CS}^{+}\right)$. Reversal learning is typically assessed using the number of sessions, trials and errors (incorrect responses on noncorrection trials) required to reach criterion. In addition, latencies, percentage of bias and perseveration score may be analyzed (see Step 10B(vi)).

Several reversal procedure parameters can be modified to affect performance and provide insight into underlying processes. One example is the use of stimuli that are harder to discriminate relative to standard shape images (e.g., stimuli that have overlapping features of both the correct and incorrect stimuli (blended, morphed or complex photographs) ${ }^{30,92}$. This may increase the potential of detecting certain experimentally induced performance improvements through the extension of the reversal training period. Alternatively, the use of luminance-matched patterns instead of shape stimuli has been observed to speed visual discrimination criterion attainment (A.C.M., J.A. and T. Robbins, unpublished data; Fig. 3d).

Post-reversal experimental manipulations include the testing of serial reversals, which may be used to supplement ${ }^{66}$ as well as to add distinct information ${ }^{61}$ to that observed for the 
Figure 4 | 5-CSRT task. (a) Schematic of a five-hole mask used in the 5-CSRT paradigm. (b) Flowchart overview of the 5-CSRT task. A trial is initiated when the subject enters and withdraws its head from the illuminated magazine. After a 5-s delay, a white square stimulus is briefly presented in one of the five response windows. Touching the white-square location either when the stimulus is present or during a short subsequent limited-hold period is recorded as a correct trial and rewarded. Collection of reward initiates a 5-s ITI. A response in any other response window is recorded as an incorrect trial and results in a time-out period, before the beginning of the 5-s ITI. Failure to respond at the screen during the stimulus presentation or the limited-hold period is recorded as an omission and also leads to a time out. Response(s) before the onset of the stimulus is recorded as a premature response, and lead to a time out, but premature trials do not contribute to the session trial count. After the ITI has elapsed, the magazine is illuminated and the subject can initiate another trial. $(\mathbf{c}, \mathbf{d})$ Representative data illustrating the dependence of response accuracy and omission, respectively, on stimulus duration in adult C57Bl/6J mice (S.R.O.N., L.M.S. and T.J.B., unpublished data). Data are means \pm s.e.m.

first reversal. They also include multiple repetitions of acquisition and reversal (with novel stimuli sets), which may reduce the amount of learning transfer from previous reversals. Both of these forms of repeated reversal learning may recruit separate or additional learning mechanisms to those used on first reversal exposure. Another post-reversal experimental manipulation is the replacement of either $\mathrm{CS}^{+}$or $\mathrm{CS}^{-}$stimuli with a novel stimulus during the reversal phase. When used in conjunction with multiple reversals, this manipulation may help dissociate processes such as perseveration toward the previously rewarded option (e.g., replacing $\mathrm{CS}^{+}$with a novel stimulus during reversal) or avoidance of the previously nonrewarded option (e.g., replacing $\mathrm{CS}^{-}$with novel stimulus), which can affect reversal learning performance. This strategy has been successfully used in a touchscreen visual discrimination and reversal paradigm in marmoset monkeys ${ }^{61}$.

Variants of the visual discrimination and reversal learning procedure using more than two stimuli and/or response windows are also in the process of development. The basic procedure for these is similar to that for the standard task. For example, a threestimulus version has been performed recently in rats (J.A., A.C.M., D. Theobald and T. Robbins, unpublished data). In lieu of using two stimuli in two locations, three different stimuli (one stimulus designated as $\mathrm{CS}^{+}$and the other two stimuli both designated as $\mathrm{CS}^{-}$) are presented across three different locations during visual discrimination learning (using a three-window mask). Following reversal, the previous $\mathrm{CS}^{+}$becomes a $\mathrm{CS}^{-}$, and one of the previous $\mathrm{CS}^{-}$stimuli becomes the new $\mathrm{CS}^{+}$. The third stimulus remains a $\mathrm{CS}^{-}$during both discrimination and reversal learning. This three-stimulus task is more difficult than the two-stimulus version and may thus provide greater opportunity for the detection of certain procognitive effects. A three-stimulus task also permits separation of errors of perseveration (i.e., responses to the previous $\mathrm{CS}^{+}$) from errors that occur using other search strategies (i.e., spatial response bias, including responses to the constant $\mathrm{CS}^{-}$). Similar effects may be observed if the constant $\mathrm{CS}^{-}$ stimulus is instead included as a neutral stimulus (e.g., recorded but not programmed consequences upon touching the neutral CS stimulus) in both discrimination and reversal phases. Another example is retaining the use of only two stimuli but increasing the number of locations to three or four (using an appropriate three- or four-window mask), thereby increasing the ratio of a
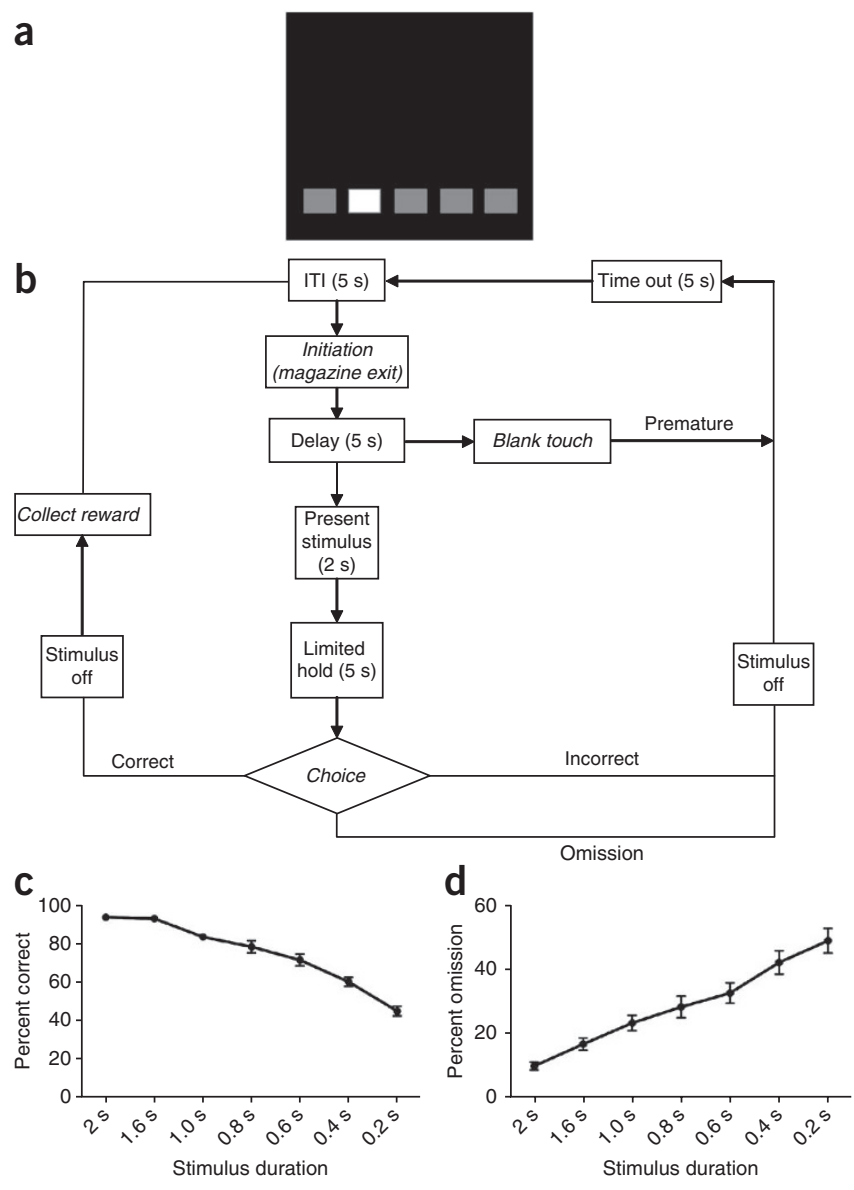

locations to stimuli. This procedure increases the difficulty of the task and helps control or rule out the possibility of subjects solving the discrimination or reversal using simple configural learning strategies (e.g., when $\mathrm{CS}^{+}$left/CS ${ }^{-}$right, touch left; when $\mathrm{CS}^{+}$right/CS- left, touch right). This may also help in reducing the development of spatial biases.

\section{5-CSRT task}

The basic 5-CSRT task requires the animal to sustain and divide its attention across a row of five screen locations to detect and respond to a brief visual stimulus (Fig. 4). Variations of the mouse touchscreen 5-CSRT task procedure outlined here have been previously described, with the main protocol featuring several key differences from the standard operant procedure in the rat ${ }^{83}$. In brief, each session consists of 40-60 trials, for which a maximum of $60 \mathrm{~min}$ is allowed, whereas, for rats, the number of trials is typically 100 -plus in 30-60 min. Each session begins with delivery of a free reward and illumination of the magazine light. Upon reward collection from the magazine, the magazine light is extinguished and a 5-s ITI period commences. After the ITI elapses, the magazine light is again illuminated to indicate that a trial can now be initiated. This 5-s ITI and second magazine entry requirement after reward collection was specifically introduced into the mouse touchscreen procedure to space trials further apart; this was done to permit mice a longer time to consume their rewards and to add a self-pacing element to help counteract difficulties that mice sometimes experience with the pace of the more traditional version (e.g., resulting in a high level of trial omissions). 
TABLE 1 | 5-CSRT task training procedure

\begin{tabular}{|c|c|c|c|c|c|c|c|c|}
\hline Step & Training stage & $\begin{array}{l}\text { Session } \\
\text { length } \\
(\mathrm{min})\end{array}$ & $\begin{array}{l}\text { Trials per } \\
\text { session (max) }\end{array}$ & Delay (s) & ITI $(s)$ & Stimulus duration (s) & Criterion & Timing \\
\hline $10 C(i-i v)$ & 5-CSRT training & 60 & $\begin{array}{l}40-60 \text { (mice), } \\
100 \text { (rats) }\end{array}$ & 5 & 5 & $\begin{array}{l}\text { Progression from } 8 \text { to } \\
4 \text { to } 2 \text { (or lower) }\end{array}$ & $\begin{array}{l}\text { Complete all } \\
\text { trials, }>80 \% \\
\text { accuracy } \\
\text { and }<20 \% \\
\text { omissions for } \\
3 \text { out of } 4 \\
\text { consecutive } \\
\text { sessions at } \\
\text { each duration }\end{array}$ & $\begin{array}{l}60 \mathrm{~min}, \\
\sim 12-30 \\
\text { sessions }\end{array}$ \\
\hline \multirow[t]{4}{*}{$10 C(v)$} & $\begin{array}{l}\text { 5-CSRT probes } \\
\text { Variable stimulus } \\
\text { durations } \\
\text { (between- } \\
\text { session) }\end{array}$ & 60 & $\begin{array}{l}40-60 \text { (mice), } \\
100 \text { (rats) }\end{array}$ & 5 & 5 & $\begin{array}{l}\text { Consecutive (e.g., } \\
\text { descending) or } \\
\text { counterbalanced } \\
\text { sessions at each } \\
\text { duration, e.g., } 2.0 \text {, } \\
1.0,0.8,0.6,0.4 \\
\text { and } 0.2\end{array}$ & NA & $\begin{array}{l}2 \text { sessions } \\
\text { per probe } \\
\text { with } 2 \\
\text { sessions } \\
\text { baseline in } \\
\text { between }\end{array}$ \\
\hline & $\begin{array}{l}\text { 5-CSRT probes } \\
\text { Variable stimulus } \\
\text { durations } \\
\text { (within-session) }\end{array}$ & 60 & $\begin{array}{l}40-60 \text { (mice), } \\
100 \text { (rats) }\end{array}$ & 5 & 5 & $\begin{array}{l}\text { Variable within- } \\
\text { session stimulus } \\
\text { durations presented in } \\
\text { pseudorandom order, } \\
\text { e.g., } 2.0,1.0,0.8 \text {, } \\
0.6,0.4 \text { and } 0.2\end{array}$ & NA & $\begin{array}{l}2 \text { sessions } \\
\text { per probe }\end{array}$ \\
\hline & $\begin{array}{l}\text { 5-CSRT probes } \\
\text { Increased } \\
\text { trials and } \\
\text { session length }\end{array}$ & 90 & $\begin{array}{l}100-200 \text { (mice), } \\
150-300 \text { (rats) }\end{array}$ & 5 & 5 & $\begin{array}{l}\text { Typically baseline } \\
\text { (e.g., 2s) }\end{array}$ & NA & $\begin{array}{l}1-2 \text { sessions } \\
\text { for baseline, } \\
2 \text { sessions } \\
\text { for probe }\end{array}$ \\
\hline & $\begin{array}{l}\text { 5-CSRT probes } \\
\text { Reducing } \\
\text { stimulus } \\
\text { brightness }\end{array}$ & 60 & $\begin{array}{l}40-60 \text { (mice), } \\
100 \text { (rats) }\end{array}$ & 5 & 5 & $\begin{array}{l}\text { Typically baseline } \\
\text { (e.g., } 2 \mathrm{~s})\end{array}$ & NA & $\begin{array}{l}1-2 \mathrm{~d} \text { for } \\
\text { baseline, } \\
2 \mathrm{~d} \text { per } \\
\text { probe }\end{array}$ \\
\hline
\end{tabular}

This additional ITI and/or initiation requirement can be removed to render the procedure more equivalent to standard versions of the 5 -CSRT $\operatorname{task}^{83}$ for use in testing rats or to increase the difficulty of the current mouse protocol.

After the 5-s ITI has elapsed, trials can be initiated by magazine head entry (signaled by turning off the magazine light and by a 0.2 -s auditory click) and exit from the reward magazine. A solid white square stimulus then appears briefly (e.g., 2 s) in one of the five screen locations after a fixed 5-s delay period. The stimulus position is chosen pseudorandomly such that it is equally presented at each location throughout the session. There are no correction trials implemented after incorrect responses or omissions. There are four possible trial outcomes. Touches to one of the screen locations during stimulus presentation or during a 5 -s limited hold time period after the stimulus is removed result in either a correct or incorrect trial. Correct trials are recorded if the response is in the same location as the visual stimulus, whereas incorrect trials are recorded if one of the other four blank locations is touched. If no touch response is made, the trial is classified as an omission. If a location is touched during the delay before stimulus onset, the trial is deemed as premature. Premature trials are recorded but are not included in the total trial count for the session (e.g., similar to correction trials in other procedures). After premature responses, the same trial is repeated until a correct, incorrect or omission trial is performed. The stimulus is removed from the screen immediately if it is still present after a correct or incorrect response. Correct trials are followed by illumination of the magazine light and delivery of a 5-s tone and a reward. Incorrect trials, premature trials and trial omissions are all followed by a 5-s time-out period in which the house light is turned on. Upon reward collection or after the time-out period has elapsed, the 5-s ITI period preceding 
the next trial commences. Baseline 5-CSRT parameters are reached by training animals through a series of stages in which the stimulus duration is gradually reduced to increase task difficulty (see Table 1). When animals are performing stably at baseline, a variety of probes can be used to further assess performance, including variations in stimulus duration, stimulus brightness, delay before stimulus presentation and the number of trials per session length. These alternatives are discussed in more detail in the PROCEDURE. Other manipulations have also been implemented in the standard operant apparatus that may also serve to increase the attentional demands of the touchscreen task. Examples include removal of the requirement for trial initiation (e.g., experimenter-paced rather than self-paced target presentation) and alteration of the rate of presentation of the stimuli (e.g., high event rate of $1 \mathrm{~s}$ or low event rate of 20 s; refs. 93,94).

\section{MATERIALS}

\section{REAGENTS}

- Rats or mice (Reagent Setup) ! CAUTION All experiments using live animals must be approved by the relevant institutional and governmental bodies and performed according to their regulations.

- Animal housing (Reagent Setup)

- Cleaning materials (e.g., TriGene, 70\% (vol/vol) ethanol solution, stiff brush)

- Rodent food pellets (e.g., rodent pellets, Special Diets Services)

- Food rewards: Either solid (e.g., Bio-Serv purified rodent dustless precision pellets, $45 \mathrm{mg}$ (rat)/14 mg (mouse), through Sandown Scientific) or liquid (Yazoo strawberry milkshake, FrieslandCampina) $\Delta$ CRITICAL Reward pellets generally work well for rats. We use either liquid or solid rewards for mice. In some cases, liquid rewards may be the better option (e.g., when using manipulations that result in motoric changes that could affect chewing, cause dry mouth or reduce motivation). Liquid rewards also afford more flexibility to vary reinforcement value (e.g., concentration, volume). I CAUTION When filling reward dispensers with dustless precision pellets, take care to discard dust, as this can potentially clog dispensers. I CAUTION All liquid reward containers and delivery lines should be thoroughly rinsed at the end of each testing day to prevent clogging and/or growth of potentially harmful microorganisms.

\section{EQUIPMENT}

- Sound- and light-attenuating boxes with ventilation system, enclosing an operant chamber and reward delivery system

- Touchscreen operant chambers (from, e.g., Campden Instruments, Med Associates, other commercial suppliers; or custom-made operant system). Note that these are species specific. Chambers made by different companies may vary, but they share many common features. The specific model used depends on the experimenter's needs and preference. In Equipment Setup we describe mouse and rat chambers from Campden Instruments and our in-house assembled boxes

- Camera above the chamber connected to closed circuit monitor and digital video-recording device to monitor and record animals' behavior (optional but recommended)

- Controlling software and devices (generally available from operant chamber supplier)

- Black plastic masks with response windows (the number and size of which differ between tasks_- see Equipment Setup)

- Shelf for rat chamber (for some tasks, see Equipment Setup)

- Appropriate data analysis software

- Personal protection equipment (e.g., disposable medical gloves, lab coat or coverall, FFP2 mask) should always be worn when handling or working near animals, to minimize allergen exposure ! CAUTION Completely power off and take care not to damage touchscreens whenever inserting or removing response window masks. Failure to do so may require touchscreen recalibration and/or touchscreen maintenance or replacement.

\section{REAGENT SETUP}

Rodents Laboratory-bred or commercially available rats/mice are generally used for testing. There are some advantages to testing male rodents, such as avoiding potential estrus cycle-related performance variability in female rodents, and potentially increased inter-male aggression when male rodents must be tested in the same apparatus as female rodents ${ }^{95,96}$. Most commonly, we use Lister Hooded rats and mice on the C57BL/6 or 129 substrain genetic backgrounds. We prefer to begin training when rodents are young adults, for example, 10- to 14-week-old mice. However, female rodents, aged rodents and various strains have also been successfully tested ${ }^{28,97}$. I CAUTION All experiments using live animals must be approved by the national and institutional bodies and performed according to their regulations. I CAUTION If animals are not fully grown when food restriction begins, they must be allowed to gain sufficient weight as they continue to grow. Standard strain growth curves are available for guidance (e.g., http://jaxmice. jax.org/support/weight/index.html). See Step 3 for further details. $\Delta$ CRITICAL We advise consulting with your institutional animal care regulatory body regarding all aspects of animal husbandry when planning and designing experiments with rodents.

Animal housing Rats and mice should be housed in groups (e.g., 2-5) with sawdust, bedding and (optional, although recommended) some form of shelter (e.g., plastic or cardboard tube). Cages should be cleaned and bedding, etc. changed weekly. The housing room should be maintained at a constant temperature $\left(20-24^{\circ} \mathrm{C}\right)$ and humidity $(55 \pm 10 \%)$. Lighting is usually on a 12-h light-dark cycle, with lights off at 7:00 a.m. or 7:00 p.m. (we favor lights off at 7:00 a.m., so that rodents can be tested in the active period of their circadian cycle).

\section{EQUIPMENT SETUP}

Campden operant chambers Housed inside a dense fiberboard box, these are equipped with a fan (for ventilation and masking extraneous noise), touchscreen monitor (rat: 15.0 inch, screen resolution $1,024 \times 768$ (rotated); mouse: 12.1 inch, screen resolution $600 \times 800)$, tone and click generator, house light (LED), magazine unit (with light and IR beam to detect entries; in the standard configuration this is outside the testing arena, on the wall opposite the touchscreen) and pellet dispenser and/or pump connected to bottles of liquid reward (Fig. 5). The chambers have a trapezoidal shape (in cm, rat: 30 high $\times 33$ long (screen-magazine) $\times 25$ wide (at screen) or 13 wide (at magazine); mouse 20 high $\times 18$ long $\times 24$ or 6 wide; excluding space between floors), which is composed of three black plastic walls opening on to the touchscreen. This is intended to help focus the animal's attention to the touchscreen and reward delivery area. The touchscreen uses optical IR sensors and therefore does not require the subject to exert any pressure for responses to be registered. We have found that this feature greatly facilitates performance compared with other types of touchscreens, and that they are particularly robust and reliable (e.g., unaffected by minor screen surface scratches or dirt that may accumulate during testing). Access to the chamber is through a transparent lid, which can be secured to the trapezoidal walls with latches during animal testing. The floor is made of perforated stainless steel, raised above a tray lined with filter paper. Two additional photobeams extend between the side walls of the arena, parallel to the screen, to detect the movement of an animal in the front (rat: $\sim 6 \mathrm{~cm}$ from the screen; mouse: $\sim 7 \mathrm{~cm}$ ) or in the rear (rat: $\sim 5 \mathrm{~cm}$ from the magazine; mouse: $\sim 3.5 \mathrm{~cm}$ ) parts of the arena. A small IR camera can be installed above the chamber to monitor animals' behavior (optional, but recommended). In rat chambers, attaching a shelf to the mask has proved to be effective at reducing impulsive responses and improving attention directed to the stimuli, by forcing the 
Figure 5 | Annotated photographs of a Campden Instruments rat touchscreen chamber. (1) Touchscreen, (2) black plastic mask covering touchscreen except for response windows, (3) black Perspex walls, (4) pellet dispenser, (5) IR beam assembly, (6) house light positioned above the chamber, (7) IR camera positioned above the chamber, (8) tone and click generator and (9) a sound/light-attenuating box with a ventilation fan fitted.

rat to rear up before making a choice ${ }^{27}$. In Campden rat chambers, for some tasks a spring-hinged shelf $(24 \mathrm{~cm}$ wide $\times 6 \mathrm{~cm}$ long $)$ can be attached $15 \mathrm{~cm}$ above the floor at a $90^{\circ}$ angle to the screen and mask. Campden Instruments provides advice on setting up the touchscreen equipment, including calibrating the touchscreen and reward dispenser.

Our in-house operant chambers Housed inside a melamine box, chambers (modified in our laboratory from Med Associates operant chambers) are equipped with a fan, IR touchscreen monitor (in $\mathrm{cm}$, rat: 29.0 high $\times 23.0$ wide; mouse: 16.0 high $\times 21.2$ wide; Craft Data), tone generator, click generator, house light $(3 \mathrm{~W})$, magazine (with light and IR beam) and pellet dispenser. As with the Campden system, the optical IR touchscreen does not require the subject to exert any pressure for touches to be registered. The chambers have a rectangular shape, consisting of a metal frame with clear Perspex walls (in cm, rat: 29 high $\times 31$ long $\times 24$ wide; mouse: 13 high $\times 25$ long $\times 19$ wide; excluding space below the floors). Access is through a hinged side wall that is secured with a latch during testing. The floor is made of stainless steel bars spaced $1 \mathrm{~cm}$ apart above a tray lined with filter paper. The magazine is equipped with a light $(3 \mathrm{~W})$ and a photocell nose-poke detector. A spring-hinged shelf $(20.5 \mathrm{~cm}$ wide $\times 6 \mathrm{~cm}$ long $)$ is also fitted in rat chambers $14.0 \mathrm{~cm}$ above the floor, at a $90^{\circ}$ angle to the screen and mask. Masks and stimuli A black plastic mask (in $\mathrm{cm}$, rat in-house: 38.7 high $\times$ 30.0 wide; rat Campden: 35.8 high $\times 28.0$ wide; mouse in-house: 11.8 high $\times$ 22.8 wide; mouse Campden: 24.3 high $\times 28.0$ wide) with response windows is fitted in front of the touchscreen to reduce accidental screen touches and to make response locations clearly identifiable from the background. These have varying numbers and sizes of response windows, depending on the task. For extinction, the mask should ideally be constructed to block the entire touchscreen except for a central response window (for mice, typically $5.0-7.1 \times 5.0-7.1 \mathrm{~cm}, 1.5 \mathrm{~cm}$ above the floor; for rats, window size can be of similar or larger dimensions, $2.5 \mathrm{~cm}$ from floor without a shelf, $16.0 \mathrm{~cm}$ above the floor if a shelf is included). Other mask types may also be used, depending on the procedure used (e.g., two-window reversal learning mask or three-window object-location paired-associates learning mask; Fig. 2a and ref. 30). The visual stimulus is typically a solid white square of similar dimensions and position as the mask response window.

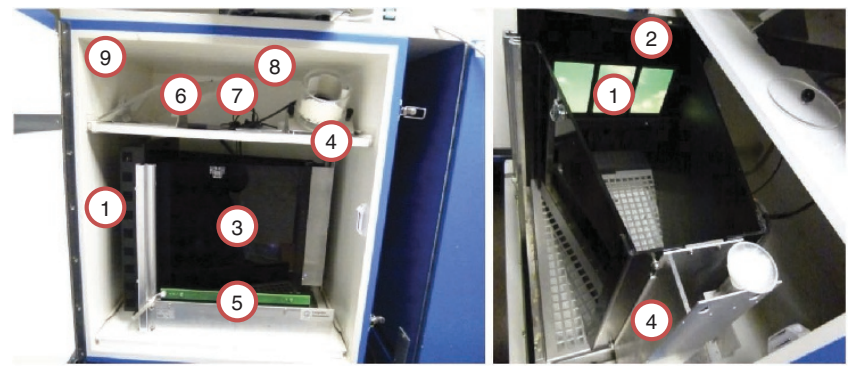

For reversal learning, a mask with two response windows is used (Fig. 3a). For mice, the two response windows are typically $7.0 \times 7.5 \mathrm{~cm}$, positioned centrally with windows $0.5 \mathrm{~cm}$ apart, $1.5 \mathrm{~cm}$ above the grid floor. For rats, the two response windows are typically $10.0 \times 10.0 \mathrm{~cm}$, positioned centrally with windows $1.0 \mathrm{~cm}$ apart, either $2.5 \mathrm{~cm}$ from floor without shelf or $16.0 \mathrm{~cm}$ above floor if shelf is included. (Other mask types might be used for reversal task variants, e.g., three-window object-location paired-associates learning mask). The visual stimuli are typically selected from a set of custommade images (e.g., Fig. 3c; ref. 30). Recent development work has demonstrated that using patterns as stimuli (e.g., Fig. 3d) can enhance the speed and extent of visual discrimination (A.C.M., J.A. and T. Robbins, unpublished data).

For the 5-CSRT task, a 5-choice mask should be inserted in front of the screen (Fig. 4a). For mice, the five response windows are typically $2.0-4.0 \times$ $2.0-4.0 \mathrm{~cm}$ squares, positioned centrally with windows spaced $1.0 \mathrm{~cm}$ apart, $1.5 \mathrm{~cm}$ above the grid floor. For rats, the five response windows are typically $2.0-3.0 \times 2.0-3.0 \mathrm{~cm}$ squares, positioned centrally with windows spaced $1.0-1.5 \mathrm{~cm}$ apart, $1.5-2.0 \mathrm{~cm}$ from the grid floor. For rats, the shelf is not used. The visual stimulus is typically a solid white square of similar dimensions and position as the mask response windows.

Controlling software and devices Controlling software can be purchased from the suppliers of the operant chambers, e.g., Whisker ${ }^{98}$ and ELO software (ELO Touchsystems). Multiple chambers may be controlled by a single computer, although it is important to check that minimum system requirements are met (e.g., memory and graphics cards) to prevent delays in stimuli presentation and chamber responses. All task software is based on earlier publications and is available (excluding, in some cases, recent modifications) with user's guides and technical support from Campden Instruments (and in some cases from Med Associates (K-Limbic) or other suppliers). Alternatively, software may be programmed using common programming languages such as Visual Basic 6.0 (Microsoft).

\section{PROCEDURE}

\section{Preparation for pretraining}

1| If it is not necessary to transport animals to the facility from an external source, you may proceed directly to Step 2. Otherwise, transported animals should be permitted an acclimatization period of at least $7 \mathrm{~d}$, with free access to food and water, before proceeding to Step 3. Begin basic handling and weighing procedures (Step 2) after $4 \mathrm{~d}$ of acclimatization. $\triangle$ CRITICAL STEP Some cohorts of mice have relatively high between-subject variability, and, thus larger sample sizes are required. Where possible, calculation of sample size should based on a power calculation derived from previous work with that strain of animal, ideally from the same animal supplier. We advise minimizing the age range of the cohort(s) tested to reduce potential age-related variability.

$\Delta$ CRITICAL STEP For touchscreen naive animals to be tested on extinction, follow preparation and pretraining Steps 1-7 before proceeding to extinction training (Step 10A). For touchscreen-naive animals to be trained on reversal learning or the 5-CSRT task, follow preparation and all pretraining Steps 1-9 before proceeding to reversal rearning training (Step 10B) or 5-CSRT training (Step 10C). If subjects have previously been trained and tested on another instrumental touchscreen task in the battery, maintain food restriction and begin training the animals at the highest pretraining step previously run. 
2| Weigh each animal for three consecutive days while they receive ad libitum food and water, and then calculate the mean free-feeding weight of each animal.

$\Delta$ CRITICAL STEP Ensure that each animal can be stably and reliably identified throughout the course of the experiment.

3| Begin food restriction, making sure to adhere to all relevant institutional animal handling guidelines. Slowly reduce (e.g., over 3-7 d) the weight of individual animals down to the goal weight, which will be a percentage of the measured free-feeding weight (e.g., we use $85-95 \%$, which is in line with our institutional guidelines) by controlling the daily amount of food they are given (e.g., for rats, $\sim 7 \mathrm{~g}$ food per $100 \mathrm{~g}$ of body weight; for mice, $\sim 2-3 \mathrm{~g}$ food per 25-35 g of body weight). Start Step 4 when animals are close to their goal weights. Maintain food restriction throughout touchscreen testing. $\triangle$ CRITICAL STEP It is important to check the weight of animals daily (mice) or twice a week (rats) until the target weight is reached. This also helps habituate the animals to being handled. Aim to avoid a weight reduction of greater than $5 \%$ per day and a weight reduction below $85 \%$ of that observed during free-feeding.

$\triangle$ CRITICAL STEP If animals are not fully grown when food restriction begins, the target weight should be adjusted upward week-by-week on the basis of known growth curves for that species or strain in order to account for additional weight gain that is sufficient to ensure normal development (see Reagent Setup).

4| Introduce reward (pellets or milkshake) inside the cage to habituate the animals for 1-3 d. Solid rewards may be scattered on the cage floor; liquid rewards should be poured into a wide, shallow dish.

\section{Pretraining}

5| Set up the apparatus (see MATERIALS) for the pretraining stage (Experimental design; stage 1), with all electronic components on so that subjects may habituate to these. In this and all subsequent steps, use the touchscreen masks and stimuli as appropriate for the task (see Equipment Setup). Although it is not necessary to run any software during stage 1, we recommend recording subjects' activity if the necessary apparatus and software is available. If the computer program does not automatically do so, manually place $\sim 10$ reward pellets or $0.2 \mathrm{ml}$ of liquid reward into the magazine of each chamber before the session. Place each rodent into its assigned chamber for $30 \mathrm{~min}$. Remove the rodent and check that all reward has been consumed. Return each animal to its respective home cage. The criterion for advancing to Step 6 is consuming all reward within a session for two consecutive sessions.

$\Delta$ CRITICAL STEP Animals achieving criterion can generally be moved to Step 6 in the subsequent testing session unless there are other experimental reasons for adjusting the pretraining progression (see Experimental design). Excessive habituation may serve to retard operant learning in some animals.

$\Delta$ CRITICAL STEP Animals require fewer standard rodent food pellets when receiving rewards during training; adjust daily food allowance as appropriate to maintain goal weight. Maintain the animal weighing routine described in Step 3. If large disparities in weights between animals within a cage exist before, or emerge during the course of, behavioral testing, consider feeding individual animals separately before returning them to their home cages to better control the daily food intake.

$\triangle$ CRITICAL STEP As the behavioral performance of animals can be influenced by changes in the experimental context, aim to train, weigh and feed each animal at approximately the same time each day. Use the same operant box for each animal and have the same experimenter or set of experimenters conduct the experiment. Attempt to counterbalance chambers and testing times across experimental groups.

$\Delta$ CRITICAL STEP Operant chambers should be cleaned regularly (e.g., once a week or more) to avoid context change during sensitive task phases, to ensure that the touchscreen and IR photobeams retain maximum sensitivity and to prevent accumulation of dirt and excrement. We typically dismantle inner chambers (as much as possible) and clean them with surface disinfectants (e.g., TriGene and 70\% (vol/vol) ethanol) and a paper towel or a stiff brush.

6| Set up the apparatus as detailed in MATERIALS and the software program for stage 2, with settings as detailed in Experimental design. For extinction using a single location, only that single location should be used to display stimuli. Place each subject in its assigned chamber and start the session. The session finishes after 60 min or 100 trials (rat) or 30 trials (mouse) are completed, whichever comes first. After session termination, return each animal to its respective home cage. Advance individual subjects to the next training phase when they have achieved a criterion of completing all 30 trials (mice) or 60 trials (rats) within the 60 -min session. Animals can be moved to Step 6 for the session immediately after achieving criterion.

$\Delta$ CRITICAL STEP Animals achieving criterion can generally be moved to Step 7 on the subsequent testing session unless there are other experimental reasons for adjusting the pretraining progression (see Experimental design). Excessive Pavlovian training exposure may serve to retard later operant learning in some animals.

$\triangle$ CRITICAL STEP At the end of each session, record crucial data for each subject (e.g., number of correct responses, number of trials completed) in case of computer malfunction. However, most software programs will record many other measures. 
$\triangle$ CRITICAL STEP If you are testing the effects of a manipulation before the onset of the experiment (see case 1 in Experimental design), ensure that the animals in the experimental and control groups complete comparable numbers of trials per session by limiting the number of trials given per session to accommodate the lowest responders.

7| Repeat the procedure in Step 6 using the software program for stage 3, with settings as detailed in Experimental design. Proceed to Step 10 for animals to be tested on extinction after they reach criterion on this step.

8| If your program does not do this automatically, manually provide a single free reward into the magazine before running the software for this step. Repeat the procedure in Step 6 using the software program for stage 4 as detailed in Experimental design.

9| Proceed as in Step 8, but by using a different software program for stage 5 (see Experimental design). The criterion for completing this stage is completing all trials with $\geq 80 \%$ correct (not including correction trials) within 60 min (rat), or with $\geq 75 \%$ correct within 35 min (mouse), on two consecutive sessions. For animals to be trained on the 5-CSRT task, if no ITI is desired within the final 5-CSRT task protocol (e.g., either to increase task difficulty or if rats are used as subjects (see Experimental design), set the ITI to $0 \mathrm{~s}$ when performing this and all pretraining steps. In addition, as no correction trials are given during 5-CSRT training (Step 10C), they may also be removed from the current step as desired.

$\triangle$ CRITICAL STEP There is likely to be variation in the number of days that animals require to complete pretraining. We suggest resting animals when they reach criterion at Step 9, with reminder sessions. All subjects can then be rebaselined so that the entire group can advance to a specific touchscreen task on the same day, unless there is a specific experimental reason to do otherwise (see Experimental design).

$\triangle$ CRITICAL STEP If subjects are scheduled to receive experimental treatments after pretraining but before task acquisition (case 2, Experimental design), perform these now (after completing Step 9). Rebaseline the animals on Step 9 (stage 5, Experimental design) before task-specific training.

$\triangle$ CRITICAL STEP Attempt to counterbalance experimental groups according to the number of sessions required to complete pretraining.

Task

10| Proceed to the extinction (option A), reversal learning (option B) or 5-CSRT task (option C).

(A) Extinction

(i) Acquisition. Begin training animals on once-daily sessions of acquisition, 5-7 d per week. Maintain the same equipment setup as during pretraining, and use the software program for this stage with settings as detailed in Experimental design. Place each subject into its assigned chamber and start the session. For mice, the session finishes either after $30 \mathrm{~min}$ or 30 trials are completed (whichever comes first). For rats, a greater number of trials per session (e.g., 60 or more) may be used. After session termination, return each animal to its respective home cage. If a shorter acquisition procedure is desired before the extinction phase, simply continue training subjects on Step 7 (pretraining stage 3, Experimental design) until criterion for this acquisition phase is reached, and then proceed to Step 10A(iii). $\Delta$ CRITICAL STEP If a multiple response window mask is used (see Equipment Setup), an additional task acquisition phase may be programmed such that touches to blank locations result in stimulus offset and a time-out period (Step 9 and pretraining stage 5 in Experimental design). Touches to the blank (i.e., containing no stimulus) response windows should be measured here and in all subsequent experimental phases. This approach can help ascertain whether animals are acquiring a specific response to the visual stimulus or simply approaching the touchscreen.

$\triangle$ CRITICAL STEP If you are testing the effects of a manipulation conducted before onset of the experiment or task acquisition (cases 1 and 2, Experimental design), ensure that animals in the experimental and control groups complete comparable numbers of trials per session throughout task acquisition.

$\Delta$ CRITICAL STEP At the end of each session, record all crucial data for each subject (e.g., number of trials completed, time required) in case of computer malfunction. However, most software programs will record many other measures.

(ii) Continuation of acquisition training. The acquisition criterion for this task is the completion of all trials in $12.5 \mathrm{~min}$ (mice) over five consecutive sessions. For rats, allow more time, e.g., 25 min. In the majority of experimental situations (e.g., cases 1 and 2), we suggest advancing subjects individually to extinction training (Step 10A(iii)) when they have attained this criterion (see Experimental design for discussion and alternatives). If subjects are scheduled to receive experimental treatments after acquisition but before Step 10A(iii) (e.g., case 3), we suggest performing these when all animals have reached the criterion once, making sure to counterbalance the control and experimental groups according to acquisition performance. Animals can subsequently be rebaselined (see Experimental design). 
(iii) Extinction. Proceed as in Step $10 \mathrm{~A}(\mathrm{i})$, but use the software program for the extinction phase as described in Experimental design. For mice, sessions terminate after a maximum of 30 trials (or $10 \mathrm{~min}$ ) have elapsed. For rats, a greater number of trials (e.g., 60 or more), and therefore more time, may be used.

(iv) Continuation of extinction training. Continue extinction training for each subject until it attains a criterion of two consecutive sessions with $\geq 77 \%$ omissions (i.e., for mice, at least 23 out of 30 trials). If postextinction experimental manipulations are not of interest, we suggest testing subjects for no fewer than ten extinction sessions so that a group extinction curve may be plotted. If they are of interest, advance subjects individually to Step 10A(v) when extinction criterion is met.

$\Delta$ CRITICAL STEP At the end of each extinction session, record crucial data for each subject (e.g., number of trials completed, number of omissions) in case of computer malfunction. Analyze these daily to ascertain if criterion has been met for each subject.

$\triangle$ CRITICAL STEP In addition to withholding food reward, the light and tone conditioned reinforcers should also be withheld, as they may be sufficient to maintain responding during extinction. Variants of the protocol can be applied to test the ability of the conditioned reinforcer stimuli to maintain responding behavior in the absence of food reward by presenting the stimuli but omitting food reward during extinction.

(v) Postextinction experimental manipulations. Several postextinction manipulations may be conducted (see Experimental design). Some published examples of reinstatement procedures are described below ${ }^{45}$. Proceed in a similar manner to Step $10 \mathrm{~A}$ (iii) but with minor modifications to the basic extinction software program as follows. For reinstatement of instrumental responding through contingent partial re-exposure of the rewarding outcomes, assess animals using a software program in which rewards and all conditioned reinforcers (e.g., tone, magazine light) are delivered immediately after appropriate touch responses over the first few trials (e.g., six) of a session, with no rewards or conditioned reinforcers over the remaining trials. For reinstatement of instrumental responding through noncontingent partial re-exposure of the rewarding outcomes, assess animals with a software program in which rewards and conditioned reinforcers are delivered with some delay (e.g., $4 \mathrm{~s}$ ) after stimulus offset over the first few trials (e.g., 6) of a session, with no rewards or conditioned reinforcers over the remaining trials. For reinstatement of instrumental responding through contingent re-exposure of only the conditioned reinforcers, assess animals with a software program in which conditioned reinforcers (e.g., tone and magazine light but no reward) are delivered immediately after appropriate touch responses across all trials of the session. For reinstatement of instrumental responding through noncontingent re-exposure of the conditioned reinforcers, assess animals with a software program in which conditioned reinforcers (e.g., tone and magazine light but no reward) are delivered with some delay (e.g., 4 s) after stimulus offset, across all trials of the session.

(vi) Data analysis. Analyze the following performance measures for acquisition and extinction phases: the number of responses (and conversely, omissions) in each extinction session; the response rate per unit of time (e.g., per minute); time taken to complete a session; the number of trials and/or sessions required to reach criterion; time taken (response latency) to respond to the stimulus; time taken (magazine latency) to retrieve the reward (acquisition) or check the food magazine (extinction); and, if applicable, reinstatement of instrumental responding (this can be determined by calculating the difference between responding during reinstatement and responding during the last extinction session).

\section{(B) Reversal learning}

(i) Visual discrimination acquisition training. When subjects are ready for task training to begin, counterbalance stimulus reward contingencies (such that approximately half of each group receives a given stimulus as $\mathrm{CS}^{+}$and the other as $\mathrm{CS}^{-}$, and the rest the reverse), according to the number of sessions required to complete pretraining. Begin training on once-daily sessions of visual discrimination acquisition, 5-7 d per week. Provide a single free reward (if your program does not do this automatically). Set up the apparatus as detailed for this task in the MATERIALS section, and set up the software program for this stage with settings as detailed in Experimental design, with reward contingencies as appropriate for each subject. Place each subject in its assigned chamber and start the session. The session finishes either after 60 min or after 100 trials (rat) or 30 trials (mouse) are completed (whichever comes first). After session termination, return each animal to its respective home cage.

$\triangle$ CRITICAL STEP Give careful consideration to the stimulus set you choose. Any novel combinations of stimuli should be pre-assessed for stimulus biases; see Experimental design and ref. 30.

$\triangle$ CRITICAL STEP Carefully monitor visual stimulus biases on the first day of testing (see Step 10B(vi)). If animals show strong stimulus biases, consider revising the stimuli.

$\triangle$ CRITICAL STEP Given that performance will be at chance at the start of training, limit sessions to 50 trials (rat) or 15 trials (mouse) in 60 min for at least two sessions. Continue until subjects can complete this in 30 min. Give each subject an even number of these reduced sessions, such that they can be combined into full 100- or 30-trial sessions for analysis. If the subject completes fewer trials than required, the missed trials may be added to the trials required in the next session (if fewer than $\sim 10$ ), or given in a new session. 
$\triangle$ CRITICAL STEP If you are testing the effects of a manipulation before the onset of the experiment or task acquisition (cases 1 and 2, see Experimental design), ensure that animals in the experimental and control groups complete comparable numbers of trials per session throughout task acquisition. Cap the number of trials given per session to accommodate the lowest responders.

$\Delta$ CRITICAL STEP At the end of each session, record crucial data for each subject (e.g., number of correct responses, number of trials completed) in case of computer malfunction. However, most software programs will record many other measures (see Experimental design).

$\Delta$ CRITICAL STEP As different strains perform differently on this task, performance criteria can be adjusted accordingly.

(ii) Continuation of acquisition training. Continue acquisition training until the acquisition criterion is reached. The acquisition criterion for this task is the completion of all trials with an accuracy of $\geq 80 \%$ (alternatively, $\geq 85 \%$, e.g., excluding correction trials) for two consecutive sessions. Reversal learning is likely to be of primary interest; thus, regardless of experimental manipulation (e.g., cases 1-4, see Experimental design), we suggest resting animals when they reach criterion, with reminder sessions. When the entire group has achieved criterion the entire group may be rebaselined before progressing to Step 10B(iii) (see Experimental design for details and alternatives). If the subjects are scheduled to receive experimental treatments after acquisition but before reversal learning (case 3, see Experimental design), perform these when all animals have reached criterion (at least) once. Counterbalance the control and experimental groups according to acquisition performance, and then rebaseline the animals.

(iii) Reversal learning. Proceed as for visual discrimination acquisition (Step 10B(i)). Note that the reward contingency in the software program should be reversed for each subject.

$\Delta$ CRITICAL STEP When subjects are challenged with a reversal of the reward contingencies, the performance levels of subjects can drop to an accuracy of $20 \%$ or less in the initial reversal sessions. These low rates of reinforcement often lead to fewer responses and lower number of completed trials per session. This can cause considerable variation in the number of completed trials per session across animals making accuracy analyses difficult. To account for this, sessions can be limited to 50 trials (rat)/15 trials (mouse) in $60 \mathrm{~min}$, for at least two sessions. Continue until subjects can complete all trials in $30 \mathrm{~min}$. Give each subject an even number of these reduced sessions, such that they can be combined into full 100- or 30-trial sessions for analysis. If the subject completes fewer trials than required, the missed trials may be added on to the trials required in the next session (if fewer than $\sim 10$ ), or given in a new session.

(iv) Continuation of reversal learning training. Continue reversal learning training for each subject until they attain a criterion of completing all trials with $\geq 80 \%$ accuracy (e.g., for mice, 24 correct responses over 30 trials), not including correction trials, for two consecutive sessions. If postreversal experimental manipulations are not of interest, we suggest testing subjects for a minimum number of sessions (e.g., 20 for mice) so that a group reversal curve may be plotted. If they are of interest, either advance subjects individually to Step $10 B(v)$ when reversal criterion is met or rest animals when they reach criterion, with reminder sessions, until the entire group has achieved criterion, and then rebaseline all animals before progressing to Step 10B(v) (see Experimental design for details and discussion of alternatives).

(v) Post-reversal experimental manipulations. Several options exist, including serial or multiple reversal learning and stimulus replacement. For serial or multiple reversal learning, after initial completion of the reversal phase (Step 10B(ii-iv)), repeat the reversal phase to criterion using the same stimuli. Such serial reversals may be used to supplement ${ }^{66}$ as well as add distinct information ${ }^{99}$ to that observed for the first reversal. Alternatively, both the discrimination and reversal (Step 10B(i-iv)) may be successively repeated using a novel set of stimuli for each tandem repeat. This latter approach may be particularly useful if within-subject manipulations (e.g., drug treatments) are of interest. Animals should be appropriately counterbalanced across reversal order and experimental treatments. For stimulus replacement, replace either the $\mathrm{CS}^{+}$or the $\mathrm{CS}^{-}$with a novel stimulus during the reversal phase. When it is performed in conjunction with multiple reversals (above), with appropriate counterbalancing, this approach may help dissociate processes such as perseveration toward the previously rewarded option or avoidance of the previously nonrewarded option that can affect reversal learning performance ${ }^{61}$ (See Experimental design).

(vi) Data analysis. Analyze performance measures for visual discrimination and reversal learning phases separately. These measures include sessions, trials, correct and incorrect responses required to reach criterion; average correct and incorrect response latencies to respond to the screen after the appearance of stimuli; average magazine latency to retrieve reward following a correct response; percentage of bias to stimuli and/or response locations (particularly in the first session for each animal); perseverative responses, expressed as the average number of correction trials per incorrect response ${ }^{100}$; and percentage accuracy, expressed as the number of correct trials divided by the total number of trials multiplied by 100 . Percentage accuracy may be in the form of an acquisition or reversal curve, if all subjects complete a certain minimum number of sessions, e.g., 5, 10 and 20. In the reversal phase, other measures can also be analyzed: number of perseverative and learning errors, split into learning phases classified using various criteria and percent 
win-stay and lose-shift 101 . For the former, in some cases, incorrect responses made before achieving $>50 \%$ correct responding in a ten-trial bin are coded as perseverative errors, whereas incorrect responses made after achieving $\leq 50 \%$ correct responses in a ten-trial bin are coded as learning errors. We generally recommend an approach that defines the perseverative and new learning phases on the basis of the chance performance as determined by binomial distribution probabilities (e.g., errors during sessions with fewer than 40 (mouse) or 100 (rat) correct trials are coded as perseverative and errors during sessions with more than 40 (mouse) or 100 (rat) correct trials are coded as new learning) ${ }^{66}$. Moreover, where feasible, a related approach based on signal detection theory can be used to divide reversal learning into three phases ${ }^{99}$. Measures of discrimination $\left(d^{\prime}\right)$ and bias $(c)$ can be calculated and compared with the normal cumulative distribution function (CDF) at two-tailed criterion values $(P<0.05)$. Error values within sessions in which $\operatorname{CDF}\left(d^{\prime}\right)<0.05$ can be coded as perseverative, values between $0.05 \leq \operatorname{CDF}\left(d^{\prime}\right) \leq 0.95$ can be coded as chance and $\operatorname{CDF}\left(d^{\prime}\right)$ values of $>0.95$ can be coded as learning. Win-stay proportion is expressed as the number of correct responses followed by a second correct response, divided by the total number of rewarded responses. Lose-shift is expressed as the proportion of incorrect responses followed by correct response divided by the total number of incorrect responses.

\section{(C) 5-CSRT task}

(i) 5-CSRT training. Begin training on once-daily sessions of 5-CSRT training, 5-7 d per week. Provide a single free reward (if your program does not do this automatically). Set up the apparatus as detailed for this task in the MATERIALS section and set up the software program for this stage with settings as detailed in Experimental design. Set the appropriate initial testing parameters (Table 1): stimulus duration, $8 \mathrm{~s}$; ITI, $5 \mathrm{~s}$ or $0 \mathrm{~s}$; limited hold, $5 \mathrm{~s}$; and time out, $5 \mathrm{~s}$. Place each subject in its assigned chamber and start the session. The session usually finishes either after $60 \mathrm{~min}$ or 100 trials (rat) or 40-60 trials (mouse) are completed (whichever comes first). After session termination, return each animal to its respective home cage.

$\Delta$ CRITICAL STEP If you are testing the effects of a manipulation before the onset of the experiment or task acquisition (cases 1 and 2, see Experimental design), ensure that the animals in the experimental and control groups complete comparable numbers of trials per session throughout 5-CSRT training. Cap the number of trials given per session to accommodate the lowest responders.

$\Delta$ CRITICAL STEP At the end of each session, record all crucial data for each subject (e.g., number of correct responses, number of trials completed) in case of computer malfunction. However, most software programs will record many other measures (see Step 10C(vi)).

$\triangle$ CRITICAL STEP As a visual signal detection task, 5-CSRT performance is sensitive to parameters that affect perception of the visual stimuli (e.g., screen brightness, contrast or glare, ambient chamber illumination, stimulus size and spacing). The precise combination of these parameters may make performance systematically more or less difficult. The performance criterion at this and later stages may thus need to be slightly modified accordingly.

$\Delta$ CRITICAL STEP Certain strains or experimental groups of animals may differ in their perceptual abilities. The performance criterion at this and later stages may thus need to be modified accordingly.

(ii) Advance 5-CSRT training stage. When individual subjects attain a criterion of completing all trials with $\geq 80 \%$ response accuracy and $\leq 20 \%$ trial omissions (with an 8-s stimulus duration), continue training as in Step $10 C(i)$, with a reduced stimulus duration of $4 \mathrm{~s}$.

(iii) Advance 5-CSRT training stage. When individual subjects attain a criterion of completing all trials with $\geq 80 \%$ response accuracy and $\leq 20 \%$ trial omissions (with a 4 -s stimulus duration), continue training as in Step $10 \mathrm{C}(\mathrm{i})$, with a reduced stimulus duration of $2 \mathrm{~s}$.

(iv) Continue 5-CSRT training. 5-CSRT training is usually conducted as a precursor to post-training probe manipulations, and thus it is important to ensure a stable level of performance at this stage. In most experimental cases, we suggest synchronizing animals before advancing to probes (see Experimental design). Therefore, when individual subjects attain a criterion of completing all trials with $\geq 80 \%$ response accuracy and $\leq 20 \%$ trial omissions (with $2 \mathrm{~s}$ stimulus duration), we suggest resting them with reminder sessions until the entire group has attained criterion (at least) once, at which time the entire group may be rebaselined before progressing to probes (see Experimental design for details and alternatives). If subjects are scheduled to receive experimental treatments after training but before probe tests (e.g., case 3, see Experimental design), perform these when all animals have attained criterion (at least) once. Counterbalance the control and experimental groups according to training performance, and then rebaseline the animals. To rebaseline, we suggest continuing training until subjects show stable performance across at least three consecutive sessions ( $\geq 80 \%$ accuracy and $\leq 20 \%$ omissions).

(v) Post-training probes. Depending on the aims of the experiment, different variations of the basic 5-CSRT procedure can be implemented to probe aspects of attention and inhibitory control. We describe some of the common manipulations of the basic task. To conduct these post-training probe tests, proceed as in Step $10 \mathrm{C}$ (iv), but make modifications to the software and/or experimental parameters as detailed below. Transient treatments may be performed in an appropriately controlled way (e.g., case 4, Latin square design, Experimental design). To assess variable stimulus durations, 
the attentional demands of the task may be challenged by reducing the stimulus duration, e.g., from $2 \mathrm{~s}$ (baseline stimulus duration) to $1.5,1,0.8,0.6,0.4$ or $0.2 \mathrm{~s}$. A between-session approach can be used wherein a single stimulus duration is presented per session (or two consecutive sessions), followed by at least two baseline sessions with a 2-s stimulus duration to ensure that stable baseline performance is reattained before the subsequent challenge sessions. These interpolated baseline sessions also serve to counteract order effects, if stimulus durations are given in sequential (e.g., descending) order, rather than in counterbalanced manner (e.g., Latin square design). Alternatively, a within-session approach can be used wherein several different stimulus durations are presented randomly across trials within a session (e.g., $0.8,0.6,0.4$ and $0.2 \mathrm{~s}$ ), with an approximately equal number of presentations of each per session. For increased trials and session length, to further tax the attentional requirements of the 5-CSRT task, use a predetermined stimulus duration (e.g., a 2-s baseline), but increase the number of trials in a session to 100-200 for mice (150-300 for rats) and extend the maximum session duration from $60 \mathrm{~min}$ to $90 \mathrm{~min}$. For variable delays, the attentional demands of the task can be increased by varying the delay before target presentation, thereby changing the predictability of target occurrence. This manipulation can also be used to observe respective alterations in response bias (e.g., trial omissions or premature responding) ${ }^{102}$. For variable delays, use a predetermined stimulus duration (e.g., a 2-s baseline), but decrease (e.g., 2-4 s) or increase (e.g., 8-10 s) the delay period before stimulus onset. As for variable stimulus duration above, variable delays can be implemented in a between-session or withinsession manner. As animals readily adapt to this manipulation, it is important to space repeated presentations of each probe session by an adequate number of baseline sessions (e.g., three or four). Reducing stimulus brightness can be used to increase attentional demand by decreasing the detectability of the stimulus. Use a predetermined stimulus duration (e.g., a 2-s baseline), but decrease the brightness of the white square stimulus. The contrast of the stimulus may also be altered to similar effect.

$\triangle$ CRITICAL STEP During probe sessions, it is important that subjects in all experimental groups complete comparable numbers of trials. Cap the number of trials given per session to accommodate the lowest responders.

(vi) Data analysis. Analyze performance measures for 5-CSRT training and probe performance, for individual sessions and/or for the average of multiple consecutive days of a particular trial type. Assess accuracy, omissions, correct and incorrect response latencies, magazine latency, premature responses, the number of sessions to reach criterion performance, attentional performance and perserverative correct and incorrect responses, as follows. Measure accuracy as percentage correct, calculated from all trials in which a response was made to a correct location, divided by the total number of both correct and incorrect trials. This measure is typically used as the main index of attentional control. Omissions are the percentage of all trials in which the animal made no response. Omissions are often used as a measure of global attentional processes but may also be sensitive to differences in sensory, motor or motivational factors. Correct and incorrect response latencies are the time between the stimulus appearing on the screen and the animal making a correct or incorrect touch response. Omitted trials are not included. These latencies are often used to assess cognitive processing speed, but they can also be influenced by motivation and motoric factors. Magazine latency is the time to collect reward after a correct response. This variable is used to assess motivation and/or motor control. Premature responses are touches to the screen during the delay period, before the appearance of the stimulus; these are typically used as to measure 'waiting' impulsivity. Record the number of sessions it takes each subject to reach the criterion performance at each stage of pretraining and for each stimulus duration presented during the 5-CSRT training. This can be particularly useful if gross deficits are seen on main 5-CSRT performance. Attentional performance across the session can be assessed by splitting the session into ten-trial blocks and calculating percentage accuracy or omissions for each block. Perseverative correct responses are additional screen touches made after a correct response, but before collecting the reward; these are typically used as to measure compulsivity. Perseverative incorrect responses are additional responses made after an incorrect response, during the time-out; these are typically used as a measure of compulsivity. ? TROUBLESHOOTING

\section{? TROUBLESHOOTING}

For general troubleshooting advice on the touchscreen operant chamber testing method, see Table 2. Animals that fail to pass pretraining or acquisition phases, possibly owing to poor motivation, impaired vision or severe motoric deficits, cannot be tested in postacquisition phases, e.g., extinction and reversal learning. Investigators may wish to set a maximum number of sessions to pass these stages (e.g., 20 sessions for Step 2, 20 sessions for Steps 3 and 4), and exclude (but note and report) animals that fail to reach performance criterion by these thresholds.

High omissions or noncompletions of all available trials in 5-CSRT training and probe sessions may indicate a lack of motivation. During training, if you suspect overfeeding may be causing low motivation, you may delay feeding the subject and retest it $2 \mathrm{~h}$ later. If the problem was indeed low motivation, performance should improve. Once you have confirmed this, be careful not to overfeed the animals again and thereby perpetuate the cycle of low motivation the following day. 
PROTOCOL

TABLE 2 | Troubleshooting table.

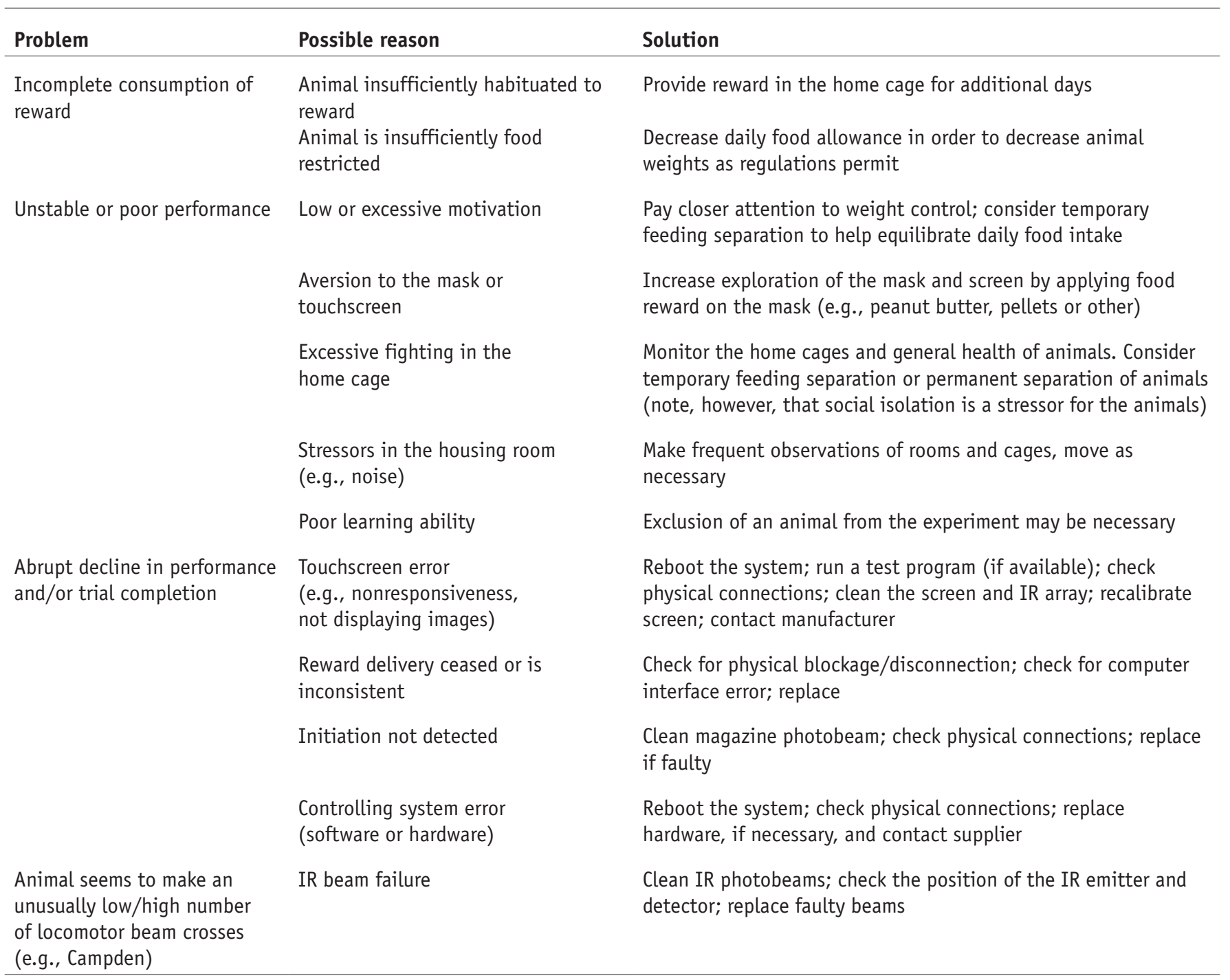

\section{TIMING}

As a rule, allow up to $\sim 80 \mathrm{~min}$ (e.g., $60 \mathrm{~min}$ of testing time plus an additional $20 \mathrm{~min}$ for transporting animals to and from the testing room, for setting up software, etc.) per testing session from Step 5 onward. Less time would be required for extinction sessions (e.g., under 30 min during acquisition (Step 10A(i-iii)), and less than 15 min is needed during extinction steps (for mice).

Approximate timing for each procedural step is indicated below (numbers of sessions). These timings are estimates based on our experience, and they reflect an average cohort of animals on each stage of the task. These estimates are based on daily training, 5-7 d per week. These estimates will vary depending on several factors, including strain, age, food deprivation and previous testing experience.

Steps 1-4, preparation for pretraining: $\sim 6$ or $10(3+7)$ d. Timing depends on whether animals are acquired from an external source, in which case a 7-d acclimatization period is required before the onset of food restriction. After acclimatization, allow for $\sim 3 \mathrm{~d}$ of initial food restriction before beginning of stage 1 pretraining. Regular handling and weighing of animals can be started $\sim 2 \mathrm{~d}$ after arrival. Reserve an average time per animal per day of $\sim 5 \mathrm{~min}$

Steps 5-9, pretraining: 10-15 sessions. Note that pretraining may take longer (e.g., 10-30 sessions) when a mask with small response windows (e.g., $<3.0 \times 3.0 \mathrm{~cm}$ ) is used and/or if rebaselining is necessary. In addition, note that full pretraining is only necessary before the first instrumental task on which an animal is tested. Before you test the animals on additional touchscreen tasks, the animals should usually be tested from the highest pretraining stage previously attained.

Being well trained, they may progress beyond this stage after relatively few sessions

Step $10 \mathrm{~A}(\mathrm{i}, \mathrm{ii})$, extinction (acquisition phase): $5-20$ sessions

Step 10A(iii,iv), extinction (extinction phase): $6-20$ sessions 
Step 10A(v), extinction (post-extinction experimental manipulations): these involve a limited number of sessions, but some manipulations may require periods of up to several weeks without testing before the probe

Step $10 \mathrm{~B}(\mathrm{i}, \mathrm{ii})$, visual discrimination acquisition: the average number of sessions required to reach acquisition criterion with standard visual stimuli is 5-6 (rat) or 8-10 (mouse). Note that additional sessions may be required if resting and rebaselining are necessary (e.g., before Step 10B(iii))

Step 10B(iii,iv), reversal learning: to reach criterion during reversal, mice typically require 400-500 trials (13-16 sessions), and Lister hooded rats typically require $\sim 600-700$ trials (6-7 sessions)

Step 10B(v), for Lister hooded rats: reversal with replacement of previous CS- typically takes 400-800 trials (4-8 sessions) to criterion; reversal with replacement of previous CS+ typically takes 300-600 trials (3-6 sessions) to criterion; serial reversal typically requires 400-600 trials (4-6 sessions) to reach criterion, but with extensive serial reversal training this timing can be reduced Step 10C(i-iv), 5-CSRT training: 12-30 sessions Step $10 C(v), 5-C S R T$ probes: rats require $\sim 1-21$ sessions. Mice may require more sessions for some of the longer training protocols (e.g., >30)

\section{ANTICIPATED RESULTS Extinction}

For the $\mathrm{C} 57 \mathrm{BL} / 6 \mathrm{~J}$ mouse strain (or mutant mice crossed to $\mathrm{C} 57 \mathrm{BL} / 6 \mathrm{~J}$ ), acquisition typically takes 5-20 sessions and extinction takes less than ten sessions on average (Fig. 2d,e and ref. 8). However, these performance profiles can vary markedly across different mouse strains. For example, another commonly used inbred strain, DBA/2J, shows much slower extinction on this task compared with the $\mathrm{C} 57 \mathrm{BL} / 6 \mathrm{~J}$ strain. These strain differences should be taken into consideration when assaying extinction in mutant mice that are not backcrossed onto a congenic $\mathrm{C} 57 \mathrm{BL} / 6 \mathrm{~J}$ background. Such differences could also be a factor when using different rat strains on this task.

\section{Reversal learning}

Figure 3 shows typical reversal performance in rats and mice. Note that performance varies depending on strain, age, stimuli used and previous testing experience.

\section{5-CSRT}

See Figure $\mathbf{4 c}, \mathbf{d}$ for examples.

\begin{abstract}
ACKNOWLEDGMENTS The protocols described here are those currently used in our laboratory and were written by current members of the group. However, many researchers have contributed to the development of touchscreen tasks and we would like to gratefully acknowledge their contribution. They include S. Bartko, J. Brigman, S. Forwood, C. Graybeal, A. Izquierdo, L. Lyon, A. Marti, K. McAllister, S. McTighe, J. Nithianantharajah, C. Romberg, J. Talpos and B. Winters. We also thank M. Hvoslef-Eide for her assistance in creating and modifying the mask schematics and flow charts. The research leading to these results has received support from the Innovative Medicine Initiative Joint Undertaking under grant agreement no. 115008, of which resources are composed of an European Federation of Pharmaceutical Industries and Associations in-kind contribution and financial contribution from the European Union's Seventh Framework Programme (FP7/2007-2013); from the Wellcome Trust/Medical Research Council (089703/Z/09/Z) and from Alzheimer's Research UK (ART/PG2006/5). A.E.H. receives funding from the European Union Seventh Framework Programme under grant agreement nos. 241995 (Project 'GENCODYS') and 242167 (Project 'SYNSYS'). J.A. was supported by the Swedish Academy of Pharmaceutical Sciences. A.E.H. was supported by the NIAAA Intramural Research Program.
\end{abstract}

AUTHOR CONTRIBUTIONS All authors contributed to the writing of this manuscript. A.C.M. coordinated this effort.

COMPETING FINANCIAL INTERESTS The authors declare competing financial interests: details are available in the online version of the paper.

Reprints and permissions information is available online at http://www.nature. com/reprints/index.html.

1. Jurado, M.B. \& Rosselli, M. The elusive nature of executive functions: a review of our current understanding. Neuropsychol. Rev. 17, 213-233 (2007).

2. Diamond, A. Executive functions. Annu. Rev. Psychol. 64, 135-168 (2013).

3. Miyake, A. et al. The unity and diversity of executive functions and their contributions to complex 'Frontal Lobe' tasks: a latent variable analysis. Cognit. Psychol. 41, 49-100 (2000).
4. Robbins, T.W. \& Arnsten, A.F.T. The neuropsychopharmacology of frontoexecutive function: monoaminergic modulation. Annu. Rev. Neurosci. 32 , 267-287 (2009).

5. Mar, A.C. \& Dalley, J.W. Cognition: attention and impulsivity. In Encycl. Behav. Neurosci. (eds. Koob, G.F., Le Moal, M. \& Thompson, R.F.) 1, 262-271 (Academic Press, 2010).

6. Roca, M. et al. Executive function and fluid intelligence after frontal lobe lesions. Brain J. Neurol. 133, 234-247 (2010).

7. Elliott, R. Executive functions and their disorders. Br. Med. Bull. 65, 49-59 (2003).

8. Holmes, A. \& Wellman, C.L. Stress-induced prefrontal reorganization and executive dysfunction in rodents. Neurosci. Biobehav. Rev. 33, 773-783 (2009).

9. Chudasama, Y. Animal models of prefrontal-executive function. Behav. Neurosci. 125, 327-343 (2011).

10. Robbins, T.W. Dissociating executive functions of the prefrontal cortex. Philos. Trans. R. Soc. Lond. B. Biol. Sci. 351, 1463-1470; discussion 1470-1471 (1996).

11. Stuss, D.T. \& Alexander, M.P. Is there a dysexecutive syndrome? Philos. Trans. R. Soc. Lond. B. Biol. Sci. 362, 901-915 (2007).

12. Miller, E.K. \& Cohen, J.D. An integrative theory of prefrontal cortex function. Annu. Rev. Neurosci. 24, 167-202 (2001).

13. Markou, A., Chiamulera, C., Geyer, M.A., Tricklebank, M. \& Steckler, T. Removing obstacles in neuroscience drug discovery: the future path for animal models. Neuropsychopharmacology 34, 74-89 (2009).

14. Chadman, K.K., Yang, M. \& Crawley, J.N. Criteria for validating mouse models of psychiatric diseases. Am. J. Med. Genet. B Neuropsychiatr. Genet. 150B, 1-11 (2009).

15. Keeler, J.F. \& Robbins, T.W. Translating cognition from animals to humans. Biochem. Pharmacol. 81, 1356-1366 (2011).

16. Homberg, J.R. Measuring behaviour in rodents: towards translational neuropsychiatric research. Behav. Brain Res. 236, 295-306 (2013).

17. Berg, E.A. A simple objective technique for measuring flexibility in thinking. J. Gen. Psychol. 39, 15-22 (1948).

18. Shallice, T. Specific impairments of planning. Philos. Trans. R. Soc. Lond. B. Biol. Sci. 298, 199-209 (1982). 
19. Stroop, R.J. Studies of interference in serial verbal reactions. J. Exp. Psychol. 18, 643-662 (1935).

20. Owen, A.M., Downes, J.J., Sahakian, B.J., Polkey, C.E. \& Robbins, T.W. Planning and spatial working memory following frontal lobe lesions in man. Neuropsychologia 28, 1021-1034 (1990).

21. Rosvold, H. et al. A continuous performance test of brain damage. J. Consult. Psychol. 20, 343-350 (1956).

22. Roberts, A.C., Robbins, T.W. \& Everitt, B.J. The effects of intradimensional and extradimensional shifts on visual discrimination learning in humans and non-human primates. Q. J. Exp. Psychol. B 40, 321-341 (1988).

23. Birrell, J.M. \& Brown, V.J. Medial frontal cortex mediates perceptual attentional set shifting in the rat. J. Neurosci. 20, 4320-4324 (2000).

24. Olton, D.S. \& Samuelson, R.J. Remembrance of places passed: spatial memory in rats. J. Exp. Psychol. Anim. Behav. Process. 2, 97-116 (1976).

25. Krechevsky, I. Antagonistic visual discrimination habits in the white rat. J. Comp. Psychol. 14, 263-277 (1932).

26. Carli, M., Robbins, T.W., Evenden, J.L. \& Everitt, B.J. Effects of lesions to ascending noradrenergic neurones on performance of a 5 -choice serial reaction task in rats; implications for theories of dorsal noradrenergic bundle function based on selective attention and arousal. Behav. Brain Res. 9, 361-380 (1983).

27. Bussey, T.J., Muir, J.L. \& Robbins, T.W. A novel automated touchscreen procedure for assessing learning in the rat using computer graphic stimuli. Neurosci. Res. Commun. 15, 103-110 (1994).

28. Bussey, T.J. et al. The touchscreen cognitive testing method for rodents: How to get the best out of your rat. Learn. Mem. 15, 516-523 (2008).

29. Bussey, T.J. et al. New translational assays for preclinical modelling of cognition in schizophrenia: the touchscreen testing method for mice and rats. Neuropharmacology 62, 1191-1203 (2012).

30. Horner, A.E. et al. The touchscreen operant platform for testing learning and memory in rats and mice. Nat. Protoc. 8, 1961-1984 (2013).

31. Pavlov, I.P. Conditioned Reflexes (Oxford University Press, 1927).

32. Bouton, M.E. Context, ambiguity, and unlearning: sources of relapse after behavioral extinction. Biol. Psychiatry 52, 976-986 (2002).

33. Myers, K.M. \& Davis, M. Mechanisms of fear extinction. Mol. Psychiatry 12, 120-150 (2007).

34. Morgan, M.A., Romanski, L.M. \& LeDoux, J.E. Extinction of emotional learning: contribution of medial prefrontal cortex. Neurosci. Lett. 163, 109-113 (1993).

35. Milad, M.R. \& Quirk, G.J. Fear extinction as a model for translational neuroscience: ten years of progress. Annu. Rev. Psychol. 63, 129-151 (2012).

36. Quirk, G.J. \& Mueller, D. Neural mechanisms of extinction learning and retrieval. Neuropsychopharmacology 33, 56-72 (2008).

37. Peters, J., Kalivas, P.W. \& Quirk, G.J. Extinction circuits for fear and addiction overlap in prefrontal cortex. Learn. Mem. 16, 279-288 (2009).

38. Robbins, T.W., Gillan, C.M., Smith, D.G., de Wit, S. \& Ersche, K.D. Neurocognitive endophenotypes of impulsivity and compulsivity: towards dimensional psychiatry. Trends Cogn. Sci. 16, 81-91 (2012).

39. Brigman, J.L. et al. Impaired discrimination learning in mice lacking the NMDA receptor NR2A subunit. Learn. Mem. 15, 50-54 (2008).

40. Karlsson, R.-M. et al. Assessment of glutamate transporter GLAST (EAAT1)deficient mice for phenotypes relevant to the negative and executive/ cognitive symptoms of schizophrenia. Neuropsychopharmacology 34 , 1578-1589 (2009).

41. Barkus, C. et al. Do GluA1-knockout mice exhibit behavioral abnormalities relevant to the negative or cognitive symptoms of schizophrenia and schizoaffective disorder? Neuropharmacology 62, 1263-1272 (2012).

42. Nithianantharajah, J. et al. Synaptic scaffold evolution generated components of vertebrate cognitive complexity. Nat. Neurosci. 16, 16-24 (2013).

43. Romberg, C., Horner, A.E., Bussey, T.J. \& Saksida, L.M. A touch screenautomated cognitive test battery reveals impaired attention, memory abnormalities, and increased response inhibition in the TgCRND8 mouse model of Alzheimer's disease. Neurobiol. Aging 34, 731-744 (2013).

44. Hefner, K. et al. Impaired fear extinction learning and cortico-amygdala circuit abnormalities in a common genetic mouse strain. J. Neurosci. $\mathbf{2 8}$ 8074-8085 (2008).

45. Lederle, L. et al. Reward-related behavioral paradigms for addiction research in the mouse: performance of common inbred strains. PlOS ONE 6, e15536 (2011).

46. Meyer, A.C. et al. Genetics of novelty seeking, amphetamine selfadministration and reinstatement using inbred rats. Genes Brain Behav. 9 790-798 (2010).
47. Lattal, K.M. \& Lattal, K.A. Facets of Pavlovian and operant extinction. Behav. Processes 90, 1-8 (2012).

48. Bouton, M.E. Context and behavioral processes in extinction. Learn. Mem. 11, 485-494 (2004).

49. Leeson, V.C. et al. Discrimination learning, reversal, and set-shifting in first-episode schizophrenia: stability over six years and specific associations with medication type and disorganization syndrome. Biol. Psychiatry 66, 586-593 (2009).

50. Cools, R., Barker, R.A., Sahakian, B.J. \& Robbins, T.W. Enhanced or impaired cognitive function in Parkinson's disease as a function of dopaminergic medication and task demands. Cereb. Cortex 11, 1136-1143 (2001).

51. Remijnse, P.L. et al. Reduced orbitofrontal-striatal activity on a reversal learning task in obsessive-compulsive disorder. Arch. Gen. Psychiatry 63, 1225-1236 (2006).

52. Dias, R., Robbins, T.W. \& Roberts, A.C. Dissociation in prefrontal cortex of affective and attentional shifts. Nature 380, 69-72 (1996).

53. Ghods-Sharifi, S., Haluk, D.M. \& Floresco, S.B. Differential effects of inactivation of the orbitofrontal cortex on strategy set-shifting and reversal learning. Neurobiol. Learn. Mem. 89, 567-573 (2008).

54. Mar, A.C., Walker, A.L.J., Theobald, D.E., Eagle, D.M. \& Robbins, T.W. Dissociable effects of lesions to orbitofrontal cortex subregions on impulsive choice in the rat. J. Neurosci. 31, 6398-6404 (2011).

55. Rolls, E.T. The functions of the orbitofrontal cortex. Brain Cogn. 55, 11-29 (2004).

56. Riceberg, J.S. \& Shapiro, M.L. Reward stability determines the contribution of orbitofrontal cortex to adaptive behavior. J. Neurosci. 32, 16402-16409 (2012).

57. Schoenbaum, G., Nugent, S.L., Saddoris, M.P. \& Setlow, B. Orbitofrontal lesions in rats impair reversal but not acquisition of go, no-go odor discriminations. Neuroreport 13, 885-890 (2002).

58. Walton, M.E., Behrens, T.E.J., Buckley, M.J., Rudebeck, P.H. \& Rushworth, M.F.S. Separable learning systems in the macaque brain and the role of orbitofrontal cortex in contingent learning. Neuron 65, 927-939 (2010).

59. Rogers, R.D., Andrews, T.C., Grasby, P.M., Brooks, D.J. \& Robbins, T.W. Contrasting cortical and subcortical activations produced by attentional-set shifting and reversal learning in humans. J. Cogn. Neurosci. 12, 142-162 (2000).

60. Rogers, R.D. et al. Tryptophan depletion impairs stimulus-reward learning while methylphenidate disrupts attentional control in healthy young adults: implications for the monoaminergic basis of impulsive behaviour. Psychopharmacology 146, 482-491 (1999).

61. Clarke, H.F., Walker, S.C., Dalley, J.W., Robbins, T.W. \& Roberts, A.C. Cognitive inflexibility after prefrontal serotonin depletion is behaviorally and neurochemically specific. Cereb. Cortex 17, 18-27 (2007).

62. Bari, A. et al. Serotonin modulates sensitivity to reward and negative feedback in a probabilistic reversal learning task in rats. Neuropsychopharmacology 35, 1290-1301 (2010).

63. Cools, R., Lewis, S.J.G., Clark, L., Barker, R.A. \& Robbins, T.W. L-DOPA disrupts activity in the nucleus accumbens during reversal learning in Parkinson's disease. Neuropsychopharmacology 32, 180-189 (2007).

64. Clarke, H.F., Hill, G.J., Robbins, T.W. \& Roberts, A.C. Dopamine, but not serotonin, regulates reversal learning in the marmoset caudate nucleus. J. Neurosci. 31, 4290-4297 (2011).

65. Clatworthy, P.L. et al. Dopamine release in dissociable striatal subregions predicts the different effects of oral methylphenidate on reversal learning and spatial working memory. J. Neurosci. 29, 4690-4696 (2009).

66. Chudasama, Y. \& Robbins, T.W. Dissociable contributions of the orbitofrontal and infralimbic cortex to Pavlovian autoshaping and discrimination reversal learning: further evidence for the functional heterogeneity of the rodent frontal cortex. J. Neurosci. 23, 8771-8780 (2003).

67. Izquierdo, A. et al. Basolateral amygdala lesions facilitate reward choices after negative feedback in rats. J. Neurosci. 33, 4105-4109 (2013).

68. Graybeal, C. et al. Paradoxical reversal learning enhancement by stress or prefrontal cortical damage: rescue with BDNF. Nat. Neurosci. 14, 1507-1509 (2011).

69. Bussey, T.J., Muir, J.L., Everitt, B.J. \& Robbins, T.W. Triple dissociation of anterior cingulate, posterior cingulate, and medial frontal cortices on visual discrimination tasks using a touchscreen testing procedure for the rat. Behav. Neurosci. 111, 920-936 (1997).

70. Kosheleff, A.R., Rodriguez, D., O'Dell, S.J., Marshall, J.F. \& Izquierdo, A. Comparison of single-dose and extended methamphetamine administration on reversal learning in rats. Psychopharmacology 224, 459-467 (2012).

71. Izquierdo, A. et al. Genetic and dopaminergic modulation of reversal learning in a touchscreen-based operant procedure for mice. Behav. Brain Res. 171, 181-188 (2006). 
72. Brigman, J.L. et al. Pharmacological or genetic inactivation of the serotonin transporter improves reversal learning in mice. Cereb. Cortex 20, 1955-1963 (2010).

73. Leonard, J.A. Five-choice serial reaction apparatus. Med. Res. Coun. Appl. Psychol. Res. Unit, 326 (1959).

74. Humby, T., Laird, F.M., Davies, W. \& Wilkinson, L.S. Visuospatial attentional functioning in mice: interactions between cholinergic manipulations and genotype. Eur. J. Neurosci. 11, 2813-2823 (1999).

75. Humby, T., Wilkinson, L. \& Dawson, G. Assaying aspects of attention and impulse control in mice using the 5 -choice serial reaction time task. Curr. Protoc. Neurosci. 8.5H.1-8.5H.15 (2005).

76. Young, J.W. et al. Nicotine improves sustained attention in mice: evidence for involvement of the 7 nicotinic acetylcholine receptor. Neuropsychopharmacology 29, 891-900 (2004).

77. Patel, S., Stolerman, I.P., Asherson, P. \& Sluyter, F. Attentional performance of $\mathrm{C} 57 \mathrm{BL} / 6$ and $\mathrm{DBA} / 2$ mice in the 5 -choice serial reaction time task. Behav. Brain Res. 170, 197-203 (2006).

78. Lambourne, S.L. et al. Impairments in impulse control in mice transgenic for the human FTDP-17 V337M mutation are exacerbated by age. Hum. Mol. Genet. 16, 1708-1719 (2007).

79. Siegel, J.A., Benice, T.S., Van Meer, P., Park, B.S. \& Raber, J. Acetylcholine receptor and behavioral deficits in mice lacking apolipoprotein E. Neurobiol. Aging 32, 75-84 (2011).

80. Yan, T.C. et al. Performance deficits of NK1 receptor knockout mice in the 5-choice serial reaction-time task: effects of d-amphetamine, stress and time of day. PloS ONE 6, e17586 (2011).

81. Fletcher, P.J., Soko, A.D. \& Higgins, G.A. Impulsive action in the 5-choice serial reaction time test in $5-\mathrm{HT}_{2 c}$ receptor null mutant mice. Psychopharmacology 226, 561-570 (2013).

82. Oliver, Y.P., Ripley, T.L. \& Stephens, D.N. Ethanol effects on impulsivity in two mouse strains: similarities to diazepam and ketamine. Psychopharmacology 204, 679-692 (2009).

83. Bari, A., Dalley, J.W. \& Robbins, T.W. The application of the 5-choice serial reaction time task for the assessment of visual attentional processes and impulse control in rats. Nat. Protoc. 3, 759-767 (2008).

84. Romberg, C., Mattson, M.P., Mughal, M.R., Bussey, T.J. \& Saksida, L.M. Impaired attention in the 3xTgAD mouse model of Alzheimer's disease: rescue by donepezil (Aricept). J. Neurosci. 31, 3500-3507 (2011).

85. Bartko, S.J. et al. Intact attentional processing but abnormal responding in M1 muscarinic receptor-deficient mice using an automated touchscreen method. Neuropharmacology 61, 1366-1378 (2011).

86. McTighe, S.M., Neal, S.J., Lin, Q., Hughes, Z.A. \& Smith, D.G. The BTBR mouse model of autism spectrum disorders has learning and attentional impairments and alterations in acetylcholine and kynurenic acid in prefrontal cortex. PloS ONE 8, e62189 (2013).

87. Oomen, C.A. et al. The touchscreen operant platform for testing working memory and pattern separation in rats and mice. Nat. Protoc. 8, 2006-2021 (2013).
88. Morton, A.J., Skillings, E., Bussey, T.J. \& Saksida, L.M. Measuring cognitive deficits in disabled mice using an automated interactive touchscreen system. Nat. Methods 3, 767 (2006).

89. Bouton, M.E., Winterbauer, N.E. \& Todd, T.P. Relapse processes after the extinction of instrumental learning: renewal, resurgence, and reacquisition. Behav. Processes 90, 130-141 (2012).

90. Bouton, M.E. \& King, D.A. Contextual control of the extinction of conditioned fear: tests for the associative value of the context. J. Exp. Psychol. Anim. Behav. Process. 9, 248-265 (1983).

91. Ji, J. \& Maren, S. Hippocampal involvement in contextual modulation of fear extinction. Hippocampus 17, 749-758 (2007).

92. Talpos, J.C., Fletcher, A.C., Circelli, C., Tricklebank, M.D. \& Dix, S.L. The pharmacological sensitivity of a touchscreen-based visual discrimination task in the rat using simple and perceptually challenging stimuli. Psychopharmacology 221, 437-449 (2012).

93. Mirza, N.R. \& Stolerman, I.P. Nicotine enhances sustained attention in the rat under specific task conditions. Psychopharmacology 138, 266-274 (1998).

94. Hahn, B., Shoaib, M. \& Stolerman, I.P. Nicotine-induced enhancement of attention in the five-choice serial reaction time task: the influence of task demands. Psychopharmacology 162, 129-137 (2002).

95. Frick, K.M. \& Berger-Sweeney, J. Spatial reference memory and neocortical neurochemistry vary with the estrous cycle in C57BL/6 mice. Behav. Neurosci. 115, 229-237 (2001).

96. Meziane, H., Ouagazzal, A.-M., Aubert, L., Wietrzych, M. \& Krezel, W. Estrous cycle effects on behavior of $\mathrm{C} 57 \mathrm{BL} / 6 \mathrm{~J}$ and BALB/cByJ female mice: implications for phenotyping strategies. Genes Brain Behav. 6, 192-200 (2007).

97. Clelland, C.D. et al. A functional role for adult hippocampal neurogenesis in spatial pattern separation. Science 325, 210-213 (2009).

98. Cardinal, R.N. \& Aitken, M.R.F. Whisker: a client-server high-performance multimedia research control system. Behav. Res. Methods 42, 1059-1071 (2010).

99. Clarke, H.F., Dalley, J.W., Crofts, H.S., Robbins, T.W. \& Roberts, A.C. Cognitive inflexibility after prefrontal serotonin depletion. Science 304, 878-880 (2004).

100. Chudasama, Y., Bussey, T.J. \& Muir, J.L. Effects of selective thalamic and prelimbic cortex lesions on two types of visual discrimination and reversal learning. Eur. J. Neurosci. 14, 1009-1020 (2001).

101. Clarke, H.F., Robbins, T.W. \& Roberts, A.C. Lesions of the medial striatum in monkeys produce perseverative impairments during reversal learning similar to those produced by lesions of the orbitofrontal cortex. J. Neurosci. 28 10972-10982 (2008).

102. Dalley, J.W., Everitt, B.J. \& Robbins, T.W. Impulsivity, compulsivity, and top-down cognitive control. Neuron 69, 680-694 (2011). 